\title{
POINTS DE HAUTEUR BORNÉE SUR LES VARIÉTÉS DE DRAPEAUX EN CARACTÉRISTIQUE FINIE
}

\author{
EMMANUEL PEYRE
}

\begin{abstract}
RÉSUMÉ. Le but de cet article est d'appliquer les travaux de Morris sur les séries d'Eisentein en caractéristique finie à l'étude asymptotique des points rationnels de hauteur bornée sur une variété de drapeaux généralisée obtenue comme quotient d'un groupe algébrique semi-simple au-dessus d'un corps global de caractéristique finie par un sous-groupe parabolique réduit. La formule obtenue pour le résidu de la fonction zêta des hauteurs a une interprétation analogue à celle connue pour un corps de nombres.
\end{abstract}

TABLE DES MATIÈRES

\begin{tabular}{|c|c|}
\hline \multicolumn{2}{|l|}{ Introduction } \\
\hline 1. Points de hauteur bornée & 2 \\
\hline 1.1. Notations & 2 \\
\hline 1.2. Métriques adéliques et hauteurs & 3 \\
\hline 1.3. Sous-variétés accumulatrices & 4 \\
\hline 1.4. Fonction zêta des hauteurs & 5 \\
\hline 2. Mesures de Tamagawa & 7 \\
\hline 2.1. Ouelques hypothèses sur les variétés & 7 \\
\hline 2.2. Ensembles de mauvaises places & 8 \\
\hline 2.3. Mesures locales & 9 \\
\hline 2.4. Lien avec les densités locales & 10 \\
\hline 2.5. Facteurs de convergence & 11 \\
\hline 2.6. Mesure de Tamagawa & 13 \\
\hline 3. Présentation du résultat & 13 \\
\hline 3.1. Facteurs $\alpha(V)$ et $\beta(V)$ & 13 \\
\hline 3.2. Expression de la constante & 15 \\
\hline 3.3. Géométrie des variétés de drapeaux généralisées & 15 \\
\hline 3.4. Hauteurs sur les variétés de drapeaux & 17 \\
\hline 3.5. Enoncé du résultat & 18 \\
\hline 4. Démonstration du résultat & 18 \\
\hline 4.1. Fonction zêta et séries d'Eisenstein & 18 \\
\hline 4.2. Ordre du pôle au sommet du cône & 20 \\
\hline 4.3. Valeur de la constante & 22 \\
\hline Références & 24 \\
\hline
\end{tabular}

\section{INTRODUCTION}

La compréhension du comportement asymptotique des points rationnels de hauteur bornée sur les variétés presque de Fano au-dessus d'un corps de nombres a fortement progressé ces dernières années notamment grâce à l'impulsion donnée par Manin (cf. [BM],

1991 Mathematics Subject Classification. primaire 14G05; secondaires 14M15. 
[FMT], [Pe1], [Sa] et [BT2]). Il serait naturel que le formalisme développé dans ce cadre s'étende dans une certaine mesure au cas des corps globaux de caractéristique finie. Il était donc tentant de chercher une formule pour le résidu de la fonction zêta des hauteurs pour les variétés de drapeaux généralisées sur un tel corps. Deux raisons motivent cet exemple; tout d'abord de telles formules asymptotiques ont été obtenues dans des cas particuliers (cf. [Se3 \$2.5 in fine], [Hs]), d'autre part le rôle joué par les travaux de Langlands dans la démonstration de ces formules asymptotiques pour les variétés de drapeaux sur un corps de nombres ([FMT], [Pe1]) peut être joué par ceux de Morris dans le cas d'un corps de fonctions global (cf. |Mo1| et |Mo2|).

Entre la première version de ce texte et sa soumission, d'autres auteurs ont fait progresser cette extension au cadre fonctionnel du programme initié par Manin. D'une part, King Fai Lai et Kit Ming Yeung dans [LY], écrit indépendamment de notre texte, se sont également intéressés aux variétés de drapeaux dans le cadre fonctionnel, sans toutefois donner d'interprétation de la constante, ce qui constitue le point crucial de notre travail. D'autre part D. Bourqui, a traité de manière complète le cas délicat des variétés toriques dans [Bou1], [Bou2] et [Bou3].

Ce texte est organisé de la façon suivante : dans la partie 1, nous fixons les notations et rappelons la définition de la fonction zêta des hauteurs, dans la partie 2 nous généralisons à la situation présente la définition de la mesure de Tamagawa associée à une métrique adélique et dans le paragraphe 3 nous énonçons le résultat dont la démonstration occupe l'ensemble de la dernière partie.

Dans un souci de complétude, nous redonnons l'ensemble des constructions, bien que la plupart des notions soient strictement analogues à celles définies sur un corps de nombres. Les premières sections de ce texte contiennent donc des redites par rapport à [Pe1].

\section{Points de haUteur BornéE}

Dans cette partie, nous transportons au cas d'un corps de fonctions global la notion de système de hauteurs connue sur un corps de nombres.

1.1. Notations. Dans la suite nous utiliserons les notations suivantes

Notations 1.1.1. Pour tout corps $F$, on note $\bar{F}$ une clôture algébrique de $F$ et $F^{s}$ la clôture séparable de $F$ dans $\bar{F}$.

Si $F$ est un corps de fonctions global, on note $\mathbf{F}_{q_{F}}$ le corps des constantes de $F$ et $M_{F}$ l'ensemble des places de $F$. Pour tout $\mathfrak{p}$ de $M_{F}$, on note $F_{\mathfrak{p}}$ le complété de $F$ en $\mathfrak{p}$ et $\mathbf{F}_{\mathfrak{p}}$ son corps résiduel. Pour toute place $\mathfrak{p}$ la norme $|\cdot|_{\mathfrak{p}}$ sur $F_{\mathfrak{p}}$ est normalisée par la relation

$$
\forall x \in F_{\mathfrak{p}}, \quad|x|_{\mathfrak{p}}=\left(\sharp \mathbf{F}_{\mathfrak{p}}\right)^{-v_{\mathfrak{p}}(x)}
$$

où $v_{\mathfrak{p}}: F_{\mathfrak{p}} \rightarrow \mathbf{Z}$ est la valuation surjective en $\mathfrak{p}$ et $\sharp A$ désigne le cardinal de $A$. On dispose donc de la formule du produit

$$
\forall x \in F, \quad \prod_{\mathfrak{p} \in M_{F}}|x|_{\mathfrak{p}}=1 .
$$

Pour toute place $\mathfrak{p}$, la mesure de Haar $\mathrm{d} x_{\mathfrak{p}}$ sur $F_{\mathfrak{p}}$ est normalisée par la relation

$$
\int_{\mathscr{O}_{\mathfrak{p}}} \mathrm{d} x_{\mathfrak{p}}=1 .
$$

On note $\mathscr{C}$ la courbe projective et lisse $\operatorname{sur} \mathbf{F}_{q_{F}}$ de corps de fonctions $F, g_{F}$ le genre de $\mathscr{C}$ et $h_{F}$ le nombre de classes de diviseurs de degré 0 sur $\mathscr{C}$. On identifiera $M_{F}$ aux points fermés de $\mathscr{C}$.

Nous omettrons $F$ dans ces notations lorsque le corps global sera indiqué par le contexte. 
Si $\mathscr{V}$ est un schéma sur le spectre d'un anneau $A$ et si $B$ est une $A$ algèbre commutative, alors $\mathscr{V}(B)$ désigne l'ensemble

$$
\operatorname{Hom}_{\operatorname{Spec} A}(\operatorname{Spec} B, \mathscr{V})
$$

et $\mathscr{V}_{B}$ le produit $\mathscr{V} \times_{\text {Spec } A} \operatorname{Spec} B$. Si $V$ est lisse sur un corps $F$, son groupe de Picard est noté Pic $V$, son groupe de Néron-Severi $\mathrm{NS}(V)$ et son faisceau canonique $\omega_{V}$. Le cône de $\operatorname{NS}(V) \otimes_{\mathbf{Z}} \mathbf{R}$ engendré par les classes de diviseurs effectifs est noté $C_{\text {eff }}(V)$. On note également $\bar{V}$ la variété $V_{\bar{F}}$ et $V^{s}=V_{F}$.

Si $n$ n'est pas divisible par la caractéristique de $F$, le faisceau étale sur $V$ des racines $n$-ièmes de l'unité est noté $\mu_{n}$. Le faisceau constant $\mathbf{Z} / n \mathbf{Z}$ est également noté $\mu_{n}^{\otimes 0}$ et pour tout entier $j$ strictement positif, $\mu_{n}^{\otimes j}$ désigne $\mu_{n}^{\otimes j-1} \otimes \mu_{n}$ et $\mu_{n}^{\otimes-j}$ le faisceau $\operatorname{Hom}\left(\mu_{n}^{\otimes j}, \mathbf{Z} / n \mathbf{Z}\right)$. Pour tout nombre premier $\ell$ distinct de la caractéristique de $F$

$$
H_{\text {êt }}^{i}\left(\bar{V}, \mathbf{Z}_{\ell}(j)\right)={\underset{\lim }{n}}_{n} H_{\text {êt }}^{i}\left(\bar{V}, \mu_{\ell^{n}}^{\otimes j}\right)
$$

et

$$
H_{\text {èt }}^{i}\left(\bar{V}, \mathbf{Q}_{\ell}(j)\right)=H_{\text {êt }}^{i}\left(\bar{V}, \mathbf{Z}_{\ell}(j)\right) \otimes \mathbf{Q}_{\ell}
$$

On note $\operatorname{Br} V$ le groupe de Brauer cohomologique de $V$ défini par

$$
\operatorname{Br} V=H_{\mathrm{ett}}^{2}\left(V, \mathbf{G}_{m}\right) \text {. }
$$

Si $V$ est une variété sur un corps global $F, V\left(\boldsymbol{A}_{F}\right)$ désigne l'espace adélique associé tel qu'il est défini par Weil dans [We, §1].

1.2. Métriques adéliques et hauteurs. La notion de métrique adélique est une généralisation immédiate à notre cadre de celle décrite dans [Pe2] pour un corps de nombres, elle-même inspirée de la notion classique de hauteur (cf. [La], [Si]).

Définition 1.2.1. Soit $V$ une variété projective, lisse et géométriquement intègre sur un corps global $F$ de caractéristique finie $p$. Soit $L$ un faisceau inversible sur $V$ et $\mathfrak{p}$ une place de $F$. Une métrique p-adique sur $L$ est une application associant à tout point $x$ de $V\left(F_{\mathfrak{p}}\right)$ une norme $\|\cdot\|_{\mathfrak{p}}$ sur la fibre $L(x)=L_{x} \otimes_{\mathscr{O}_{V, x}} F_{\mathfrak{p}}$ de sorte que pour toute section $s$ de $L$ définie sur un ouvert de $V$ l'application

$$
x \mapsto\|s(x)\|_{\mathfrak{p}}
$$

soit continue pour la topologie $\mathfrak{p}$-adique.

Si p est une place de $F$ et $\mathscr{V}$ un modèle projectif et lisse de $V$ sur $\mathscr{O}_{\mathfrak{p}}$, alors à tout modèle $\mathscr{L}$ de $L$ sur $\mathscr{V}$ on peut associer une métrique $\mathfrak{p}$-adique $\|\cdot\|_{\mathfrak{p}}$ sur $L$ qui est construite de la manière suivante : $V$ étant projective, tout point $x$ de $V\left(F_{\mathfrak{p}}\right)$ définit un point $\tilde{x}$ de $\mathscr{V}$ et $\tilde{x}^{*}(\mathscr{L})$ définit une $\mathscr{O}_{\mathfrak{p}}$-structure sur $L(x)$ dont on choisit un générateur $y_{0} ;\|\cdot\|_{\mathfrak{p}}$ est alors donnée par la formule

$$
\forall y \in L, \quad\|y\|_{\mathfrak{p}}=\left|\frac{y}{y_{0}}\right|_{\mathfrak{p}} .
$$

Une famille de métriques $\left(\|\cdot\|_{\mathfrak{p}}\right)_{\mathfrak{p} \in M_{F}}$ sur $L$ est appelée métrique adélique s'il existe un sous-ensemble fini $S$ de $M_{F}$, un modèle projectif et lisse $\mathscr{V} \operatorname{de} V$ sur le complémentaire de $S$ dans $\mathscr{C}$ et un modèle $\mathscr{L}$ de $L$ sur cet espace de sorte que pour toute place $\mathfrak{p}$ de $M_{F}-S$ la métrique $\|\cdot\|_{\mathfrak{p}}$ soit définie par $\mathscr{L} \otimes_{\mathscr{O}_{\mathcal{V}}} \mathscr{O}_{\mathfrak{p}}$.

Par abus de langage, nous appellerons hauteur d'Arakelov sur $V$ la donnée d'une paire $H=\left(L,\left(\|\cdot\|_{\mathfrak{p}}\right)_{\mathfrak{p} \in M_{F}}\right)$ où $L$ est un faisceau inversible sur $V$ et $\left(\|\cdot\|_{\mathfrak{p}}\right)_{\mathfrak{p} \in M_{F}}$ une métrique adélique sur ce fibré. Pour tout point rationnel $x$ de $V$ la hauteur de $x$ relativement à $H$ est alors définie par

$$
H(x)=\prod_{\mathfrak{p} \in M_{F}}\|\boldsymbol{s}(x)\|_{\mathfrak{p}}^{-1}
$$

où $s$ est une section de $L$ sur un voisinage de $x$, non nulle en $x$. 
On note $\mathscr{H}(V)$ l'ensemble des classes d'isomorphisme de hauteurs d'Arakelov modulo la relation d'équivalence $\sim$ engendrée par les relations de la forme

$$
\left(L,\left(\|\cdot\|_{\mathfrak{p}}\right)_{\mathfrak{p} \in M_{F}}\right) \sim\left(L,\left(\lambda_{\mathfrak{p}}\|\cdot\|_{\mathfrak{p}}\right)_{\mathfrak{p} \in M_{F}}\right)
$$

pour toute famille $\left(\lambda_{\mathfrak{p}}\right)_{\mathfrak{p} \in M_{F}}$ de nombres réels strictement positifs vérifiant $\prod_{\mathfrak{p} \in M_{F}} \lambda_{\mathfrak{p}}=$ 1. Si $x$ est un point rationnel et $H$ une hauteur d'Arakelov, $H(x)$ ne dépend que de la classe de $H$ dans $\mathscr{H}(V)$.

On dispose d'un morphisme d'oubli

$$
\boldsymbol{o}: \mathscr{H}(V) \rightarrow \mathrm{NS}(V) .
$$

On appelle système de hauteurs une section $\boldsymbol{H}$ de l'application d'oubli. Un système de hauteurs $\boldsymbol{H}$ sur $V$ induit un accouplement

$$
\boldsymbol{H}: \mathrm{NS}(V) \otimes \mathbf{C} \times V(F) \rightarrow \mathbf{C}
$$

qui est l'exponentielle d'une fonction linéaire en la première variable et telle que

$$
\forall L \in \mathrm{NS}(V), \quad \forall x \in V(F), \quad \boldsymbol{H}(L, x)=\boldsymbol{H}(L)(x) .
$$

Nous renvoyons à [Pe3] pour des exemples de hauteurs et de systèmes de hauteurs. En particulier pour tout morphisme $\phi: V \rightarrow W$ de variétés on a un diagramme commutatif

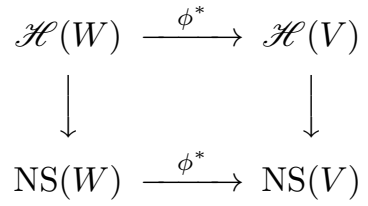

et si $E / F$ est une extension de corps on dispose d'un morphisme de normes

$$
N_{E / F}: \mathscr{H}\left(V_{E}\right) \rightarrow \mathscr{H}(V) \text {. }
$$

Si $\boldsymbol{H}_{E}$ est un système de hauteurs sur $V_{E}$ la hauteur induite $\boldsymbol{H}_{F}$ est définie par le diagramme commutatif

où $N=[E: F]$.

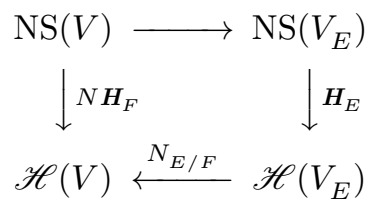

1.3. Sous-variétés accumulatrices. Comme dans le cas des corps de nombres deux notions de sous-variétés accumulatrices apparaissent naturellement.

Définition 1.3.1. Soit $H$ une hauteur d'Arakelov sur une variété projective lisse et géométriquement intègre $V$ au-dessus d'un corps global $F$ de caractéristique $p$. Pour tout sous-espace localement fermé $W$ de $V$ et tout nombre réel strictement positif $B$, on pose

$$
n_{W, H}(B)=\sharp\{x \in W(F) \mid H(x) \leqslant B\} .
$$

Remarque 1.3.1. si $H=\left(L,\left(\|\cdot\|_{\mathfrak{p}}\right)_{\mathfrak{p} \in M_{F}}\right)$ avec $[L]$ appartenant à l'intérieur de $C_{\text {eff }}(V)$, alors il existe un ouvert non vide $U$ de $V$ tel que $n_{U, H}(B)$ soit fini pour tout nombre réel $B$.

Définition 1.3.2. On note pour tout sous-espace localement fermé $W$ de $V$

$$
a_{W}(L)=\varlimsup_{B \rightarrow+\infty} \log \left(n_{W, H}(B)\right) / \log (B) \leqslant+\infty .
$$

Un fermé strict $K$ de $V$ est dit $L$-accumulateur au sens strict si et seulement si pour tout ouvert non vide $W$ de $K$, il existe un ouvert non vide $U$ de $V$ avec

$$
a_{W}(L)>a_{U}(L)
$$


Un fermé strict $K$ de $V$ est dit modérément accumulateur pour $H$ si et seulement si, pour tout ouvert non vide $W$ de $K$, il existe un ouvert non vide $U$ de $V$ tel que

$$
\left.\varlimsup_{B \rightarrow+\infty} n_{W, H}(B)\right) / n_{U, H}(B)>0 .
$$

1.4. Fonction zêta des hauteurs. Contrairement au cas des corps de nombres, les métriques étant à valeur dans $q^{\mathbf{Z}}$ sauf pour un nombre fini d'entre elles, $n_{U, H}(B)$ n'est plus asymptotiquement équivalent à une fonction de la forme $C B^{a}(\log B)^{b-1}$. Toutefois il est bien connu que l'objet naturel dans le cadre fonctionnel est la fonction zêta associée, et nous verrons plus loin qu'il est effectivement possible d'étendre à ce cadre les propriétés connues sur les corps de nombres. De manière plus précise, les travaux de Batyrev, Franke, Manin et Tschinkel, ([FMT] §2], [BT1] et [BT3]) ont soulignés l'intérêt de considérer les fonctions zêtas associées a un système de hauteurs et définies sur un ouvert du produit $\mathrm{NS}(V) \otimes$ C. Nous en rappelons maintenant la définition.

Définitions 1.4.1. Soit $\boldsymbol{H}$ un système de hauteurs sur une variété projective lisse et géométriquement intègre $V$ au-dessus d'un corps global $F$ de caractéristique $p$. Soit $U$ un ouvert de $V$. La fonction zêta associée est définie par la série

$$
\zeta_{U, \boldsymbol{H}}(s)=\sum_{x \in U(F)} \boldsymbol{H}(s, x)^{-1}
$$

où $s$ désigne un élément de $\mathrm{NS}(V) \otimes \mathbf{C}$ pour lequel la série converge absolument.

Nous réunissons maintenant quelques assertions sur le domaine de convergence des fonctions zêta. Ces assertions sont bien connues dans le cas de corps de nombres.

Lemme 1.4.1. L'ensemble sur lequel $\zeta_{U, \boldsymbol{H}}$ converge absolument est un ensemble convexe de $\mathrm{NS}(V) \otimes \mathbf{C}$ et si $s_{0}$ appartient à cet ensemble alors celui-ci contient

$$
s_{0}+i \mathrm{NS}(V) \otimes \mathbf{C} .
$$

Démonstration. Ces assertions sont des propriétés générales des séries zêta. La convexité résulte de l'inégalité de Hölder : en effet si $\zeta_{U, H}$ converge absolument pour

$$
s_{0}, s_{1} \in \mathrm{NS}(V) \otimes \mathbf{C}
$$

et si $\lambda_{0}, \lambda_{1} \in \mathbf{R}_{>0}$ avec $\lambda_{0}+\lambda_{1}=1$, alors pour tout sous-ensemble fini $I$ de $U(F)$, on a

$$
\begin{aligned}
\sum_{x \in I}\left|\boldsymbol{H}\left(\lambda_{0} s_{0}+\lambda_{1} s_{1}\right)\right|^{-1} & =\sum_{x \in I}\left|\boldsymbol{H}\left(s_{0}, x\right)\right|^{-\lambda_{0}}\left|\boldsymbol{H}\left(s_{1}, x\right)\right|^{-\lambda_{1}} \\
& \leqslant\left(\sum_{i \in I}\left|\boldsymbol{H}\left(s_{0}, x\right)\right|^{-1}\right)^{\lambda_{0}} \leqslant\left(\sum_{i \in I}\left|\boldsymbol{H}\left(s_{1}, x\right)\right|^{-1}\right)^{\lambda_{1}} .
\end{aligned}
$$

La seconde assertion résulte du fait que pour tout $x$ de $V(F)$ on a

$$
\forall s_{0} \in \mathrm{NS}(V) \otimes \mathbf{C}, \quad \forall s_{1} \in i \mathrm{NS}(V) \otimes \mathbf{R}, \quad\left|\boldsymbol{H}\left(s_{0}+s_{1}, x\right)\right|=\left|\boldsymbol{H}\left(s_{0}, x\right)\right| .
$$

Lemme 1.4.2. Si $\operatorname{Pic}(\bar{V})$ est de rang fini et si $C_{\mathrm{eff}}(V)$ est un cône polyhédrique rationnel, c'est-à-dire s'il existe $n_{1}, \ldots, n_{m}$ dans $\mathrm{NS}(V)$ tels que

$$
C_{\text {eff }}(V)=\sum_{i=1}^{m} \mathbf{R}_{\geqslant 0} n_{i}
$$

alors il existe un ouvert dense $U$ de $V$ tel que pour tout $s$ de $\mathrm{NS}(V) \otimes \mathbf{C}$ en lequel $\zeta_{U, \boldsymbol{H}}$ converge absolument, $s+C_{\mathrm{eff}}(V)$ est contenu dans le domaine de convergence absolu de $\zeta_{U, H} \cdot$ 
Démonstration. Fixons des diviseurs effectifs $D_{1}, \ldots, D_{m}$ tels que

$$
C_{\text {eff }}(V)=\sum_{i=1}^{m} \mathbf{R}_{\geqslant 0}\left[D_{i}\right]
$$

et posons

$$
U=V-\cup_{i=1}^{m} \operatorname{Supp} D_{i}
$$

Soit $s_{i}$ une section de $\left[D_{i}\right]$ correspondant à $D_{i}$ pour $1 \leqslant i \leqslant m$. Soient $s$ un élément de $\mathrm{NS}(V) \otimes \mathbf{C}$ en lequel $\zeta_{U, \boldsymbol{H}}$ converge absolument et $s^{\prime}$ un élément de $C_{\text {eff }}(V) \cap \mathrm{NS}(V) \otimes \mathbf{Q}$. Il existe un entier $\lambda$ strictement positif tel que $\lambda s^{\prime}$ puisse se mettre sous la forme

$$
\lambda s^{\prime}=\sum_{i=1}^{m} n_{i}\left[D_{i}\right]
$$

avec $n_{i} \in \mathbf{Z}_{\geqslant 0}$ pour $1 \geqslant i \geqslant m$. Soit $\left(L,\left(\|\cdot\|_{\mathfrak{p}}\right)_{\mathfrak{p} \in M_{F}}\right)$ un représentant de $\boldsymbol{H}\left(\sum_{i=1}^{m} n_{i}\left[D_{i}\right]\right)$. Comme $\operatorname{Pic}(\bar{V})$ est de rang fini, on peut supposer, quite à augmenter $\lambda$, que la classe de $L$ coïncide avec celle de $\sum_{i=1}^{m} n_{i}\left[D_{i}\right]$ dans Pic $V$. Le produit tensoriel $\bigotimes_{i=1}^{m} s_{i}^{n_{i}}$ définit alors une section $s$ de $L$. Pour tout place $\mathfrak{p}$ de $F$, la fonction

$$
\begin{aligned}
V\left(F_{\mathfrak{p}}\right) & \rightarrow \mathbf{R}_{\geqslant 0} \\
x & \mapsto\|\boldsymbol{s}(x)\|_{\mathfrak{p}}
\end{aligned}
$$

est continue et admet un maximum $B_{\mathfrak{p}}$. En outre si $S$ est une partie finie de $M_{F}$ et $\mathscr{V}$ (resp. $\mathscr{L}$ ) un modèle de $V$ (resp. $L$ ) sur $\mathscr{C}-S$ de sorte que les mestriques $\|\cdot\|_{\mathfrak{p}}$ soient définies par $\mathscr{L}$ en-dehors de $S$; alors, quitte à augmenter $S$, on peut supposer que $s$ provient d'une section $\tilde{s}$ de $\mathscr{L}$. Pour toute place $\mathfrak{p}$ dans $M_{F}-S$, tout point $x$ de $V\left(F_{\mathfrak{p}}\right)$ correspondant à un point $\tilde{x}$ de $\mathscr{V}\left(\mathscr{O}_{\mathfrak{p}}\right)$, soit $y_{0}$ un générateur de $\tilde{x}^{*}(\mathscr{L})$. On a alors la relation

$$
\|s(x)\|_{\mathfrak{p}}=\left|\frac{\boldsymbol{s}(x)}{y_{0}}\right|_{\mathfrak{p}}
$$

mais, comme $s$ provient de $\tilde{s}$, on a que $s(x)$ appartient à $u_{0} \mathscr{O}_{\mathfrak{p}}$. Donc $B_{\mathfrak{p}} \leqslant 1$.

Si $U(F)=\emptyset$, alors le résultat est évident. Dans la cas contraire, soit $x \in U(F)$ pour presque toute place $\mathfrak{p}$ de $F$ on a $\|s(x)\|_{\mathfrak{p}}=1$, par conséquent $B_{\mathfrak{p}}=1$ pour presque toute place $\mathfrak{p}$ de $F$. Mais alors

$$
\forall x \in U(F), \quad \boldsymbol{H}\left(\lambda s^{\prime}, x\right)=\prod_{\mathfrak{p} \in M_{F}}\|s(x)\|_{\mathfrak{p}}^{-1} \geqslant \prod_{\mathfrak{p} \in M_{F}} B_{\mathfrak{p}}^{-1}>0 .
$$

Autrement dit la fonction $x \mapsto \boldsymbol{H}\left(\lambda s^{\prime}, x\right)$ est uniformément minorée sur $U(F)$. On obtient

$$
\begin{aligned}
\sum_{x \in U(F)}\left|\boldsymbol{H}\left(s+\lambda s^{\prime}\right)\right|^{-1} & \leqslant \sum_{x \in U(F)}|\boldsymbol{H}(s, x)|^{-1} \boldsymbol{H}\left(\lambda s^{\prime}, x\right)^{-1} \\
& \geqslant\left(\prod_{\mathfrak{p} \in M_{F}} B_{\mathfrak{p}}\right) \zeta_{U, \boldsymbol{H}}(s) .
\end{aligned}
$$

La série définissant $\zeta_{U, H}$ converge donc absolument en tout point de

$$
s+C_{\text {eff }}(V) \cap \mathrm{NS}(V) \otimes \mathbf{Q}
$$

mais l'enveloppe convexe de ce cône est $s+C_{\text {eff }}(V)$ et le résultat découle du lemme précédent.

Lemme 1.4.3. Si $L$ est un faisceau inversible très ample sur $V$ et $N$ la dimension de $\Gamma(V, L)$, alors pour toute hauteur $H$ de la forme $\left(L,\left(\|\cdot\|_{\mathfrak{p}}\right)_{\mathfrak{p} \in M_{F}}\right)$ et tout $\epsilon>0$ la série $\sum_{x \in V(F)} H(x)^{-N-\epsilon}$ converge. 
Démonstration. Soit $s_{1}, \ldots, s_{N}$ une base de $\Gamma(V, L)$. Le système de métriques définie par

$$
\forall \mathfrak{p} \in M_{F}, \quad \forall x \in V\left(F_{\mathfrak{p}}\right), \quad \forall y \in L(x), \quad\|y\|_{\mathfrak{p}}^{\prime}=\sup _{\substack{1 \leqslant i<n \\ s_{i}(x) \neq 0}}\left|\frac{y}{s_{i}(x)}\right|_{\mathfrak{p}}
$$

est une métrique adélique sur $L$. On note $H^{\prime}$ la hauteur $\left(L,\left(\|\cdot\|_{\mathfrak{p}}^{\prime}\right)_{\mathfrak{p} \in M_{F}}\right)$. Pour presque toute place $\mathfrak{p} \operatorname{de} F,\|\cdot\|_{\mathfrak{p}}^{\prime}$ coïncide avec $\|\cdot\|_{\mathfrak{p}}$. Il existe donc une constante $C>0$ telle que

$$
\forall x \in V(F), \quad H(x) \geqslant C H^{\prime}(x) .
$$

Il suffit donc de montrer le résultat pour $H^{\prime}$. Mais

$$
\sum_{x \in V(F)} H^{\prime}(x)^{-N-\epsilon} \leqslant \sum_{x \in \mathbf{P}^{N-1}(F)} H_{\mathbf{P}^{N-1}}(x)^{-N-\epsilon}
$$

où $H_{\mathbf{P}^{N-1}}$ est la hauteur usuelle sur $\mathbf{P}^{N-1}$. Or il résulta de [Se3] theorem, p. 19] qu'il existe une constante $C$ telle que

$$
\sharp\left\{x \in \mathbf{P}^{N-1}(F) \mid H_{\mathbf{P}^{N-1}}(x)=q^{d}\right\}<C\left(q^{d}\right)^{N}
$$

Par conséquent

$$
\sum_{x \in \mathbf{P}^{N-1}(F)} H_{\mathbf{P}^{N-1}}(x)^{-N-\epsilon} \leqslant C \sum_{d}\left(q^{d}\right)^{-N-\epsilon} q^{d N}
$$

Pour terminer, nous allons énoncer une condition qui implique trivialement la périodicité la fonction zêta des hauteurs

Définition 1.4.2. Dans la suite, nous dirons que le système de hauteurs $\boldsymbol{H}$ vérifie la condition (P) si et seulement si pour tout élément $L$ de $\mathrm{NS}(V)$, il existe un représentant $\left(L,\left(\|\cdot\|_{\mathfrak{p}}\right)_{\mathfrak{p} \in M_{F}}\right)$ de $\boldsymbol{H}(L)$ dont les métriques sont toutes à valeurs dans $q^{\mathbf{Z}}$.

Remarque 1.4.4. Il résulte des définitions que les fonctions $\zeta_{U, \boldsymbol{H}}$ sont alors périodiques de groupe de périodes contenant $2 i \pi / \log (q) \mathrm{NS}(V)$.

\section{Mesures de Tamagawa}

2.1. Quelques hypothèses sur les variétés. Dans ce paragraphe, nous allons préciser quelques hypothèses sur les variétés qui nous permettront de définir la mesure de Tamagawa associée à une métrique adélique sur le faisceau anticanonique. Les hypothèses géométriques sont automatiquement vérifiées par une variété de Fano sur un corps de caractéristique 0. En l'absence d'un théorème d'annulation de Kodaira cela n'est toutefois plus le cas en caractéristique finie.

Hypothèses géométriques 1 . Dans la suite $V$ désigne une variété projective lisse et géométriquement intègre sur un corps global $F$ de caractéristique $p$ finie vérifiant les assertions suivantes :

(i) la classe du faisceau anticanonique $\omega_{V}^{-1}$ dans $\mathrm{NS}(V) \otimes \mathbf{R}$ appartient à l'intérieur du cône $C_{\text {eff }}(V)$,

(ii) les groupes de cohomologie $H^{i}\left(V, \mathscr{O}_{V}\right)$ sont nuls pour $i=1$ ou 2 ,

(iii) le groupe $\operatorname{Pic}\left(V^{s}\right)$ est un $\mathbf{Z}$-module libre de rang fini et coïncide avec $\operatorname{Pic} \bar{V}$,

(iv) le cône $C_{\text {eff }}(\bar{V})$ est polyédrique rationnel, c'est-à-dire qu'il existe $m_{1}, \ldots, m_{r}$ dans $\operatorname{Pic}(\bar{V})$ tels que

$$
C_{\text {eff }}(\bar{V})=\sum_{i=1}^{r} m_{i}
$$

(v) si $\ell$ est un nombre premier distinct de $p$, la partie $\ell$-primaire de $\operatorname{Br} \bar{V}$ est finie. 
Remarque 2.1.1. Si $V$ est $F^{s}$-rationnelle alors la première assertion de (iii) et (V) sont vérifiées. En effet, par [CTS appendice 2.A], si $V$ est $F^{s}$-rationnelle alors $\operatorname{Pic}\left(V^{s}\right)$ est stablement isomorphe à $\mathbf{Z}$ et donc libre de rang fini sur $\mathbf{Z}$ et par [Gr2, corollaire 7.5], la partie $\ell$-primaire du groupe de Brauer esy un invariant birationnel des variétés propres et lisses, ce qui implique la trivialité de ce groupe si $V$ est $F^{s}$-rationnelle.

Dans la suite nous supposerons également que la variété $V$ vérifie la condition suivante :

Hypothèse arithmétique 2. L'ensemble $V(F)$ des points rationnels de $V$ est dense pour la topologie de Zariski.

2.2. Ensembles de mauvaises places. Comme dans le cas des corps de nombres, les facteurs de convergence qui apparaissent naturellement sont les facteurs locaux de la fonction $L$ associée au groupe de Picard géométrique de la variété. Le choix de ces facteurs est justifié par les arguments de descente qui les font apparaître dans le passage aux torseurs universels (cf. [Sa] et [Pe2]). Mais pour montrer la convergence des mesures de Tamagawa, il faut comparer ces facteurs locaux à ceux donnés par le deuxième groupe de cohomologie $\ell$-adique, ce qui est rendu possible par le lemme suivant :

Lemme 2.2.1. Sous les hypothèses du paragraphe 2.1 il existe un ensemble fini de places $S$ et un modèle projectif et lisse $\mathscr{V}$ de $V$ sur $\mathscr{C}-S$ dont les fibres sont géométriquement intègres et tel que pour toute place $\mathfrak{p}$ en-dehors de $S$,

- Il existe un isomorphisme de $\operatorname{Pic}(\bar{V}) \operatorname{sur} \operatorname{Pic}\left(\mathscr{V}_{\overline{\mathbf{F}}_{\mathrm{p}}}\right)$ compatible avec les actions des groupes de Galois,

- Pour tout nombre premier $\ell$ distinct de $p$, la partie $\ell$-primaire du groupe $\operatorname{Br}\left(\mathscr{V}_{\overline{\mathbf{F}}_{\mathfrak{p}}}\right)$ soit finie.

Démonstration. La variété $V$ étant projective, on la plonge dans un espace projectif $\mathbf{P}_{F}^{N}$. Soit $\mathscr{V}$ l'adhérence de $V$ dans $\mathbf{P}_{\mathscr{C}}^{N}$. Comme $V$ est lisse et géométriquement intègre, il existe par [EGA IV 6.8.7 et 9.7.7] un ensemble fini $S$ de places tel que $\mathscr{V} \times \mathscr{C} \mathscr{C}-S$ soit lisse à fibres géométriquement intègres sur $\mathscr{C}-S$.

Par hypothèse, $\operatorname{Pic}(\bar{V})$ est un $\mathbf{Z}$-module libre de type fini qui coïncide avec $\operatorname{Pic}\left(V^{s}\right)$. Il existe donc une extension galoisienne finie $E$ de $F$ telle que $V(E) \neq \emptyset$ et

$$
\operatorname{Pic}\left(V_{E}\right) \stackrel{\sim}{\longrightarrow} \operatorname{Pic}(\bar{V}) \text {. }
$$

On ajoute à $S$ les places qui se ramifient dans l'extension $E / F$ et on note $S_{E}$ l'ensemble des places de $E$ au-dessus de $S$. Soit $\mathfrak{P}$ une place de $E$ en-dehors de $S_{E}$, soit $\widehat{\mathscr{O}_{\mathfrak{P}}^{\text {hs }}}$ le complété d'un hensélisé strict de $\mathscr{O}_{\mathfrak{P}}$ et $\operatorname{Fr}\left(\widehat{\mathscr{O}_{\mathfrak{P}}^{\text {hs }}}\right)$ son corps des fractions. Le schéma $\mathscr{V}_{\widehat{\mathscr{O}_{\mathfrak{P}}}}$ étant lisse, l'application canonique

$$
\operatorname{Pic}\left(\mathscr{V}_{\widehat{\mathscr{O}_{\mathfrak{P}}^{\text {hs }}}}\right) \rightarrow \operatorname{Pic}\left(V_{\operatorname{Fr}\left(\widehat{\mathscr{O}_{\mathfrak{P}}^{\text {hs }}}\right.}\right)
$$

est un isomorphisme. Comme, par hypothèse, $\operatorname{Pic}\left(V_{E}\right)$ est isomorphe à $\operatorname{Pic}(\bar{V})$ et donc à $\mathrm{NS}(\bar{V})$ et que le groupe de Néron-Severi ne change pas par extension de corps algébriquement clos, l'application

$$
\operatorname{Pic}\left(V_{E}\right) \rightarrow \operatorname{Pic}\left(V_{\operatorname{Fr}\left(\widehat{\mathscr{C}_{\mathfrak{P}}^{\text {hs }}}\right)}\right)
$$

est également un isomorphisme. Considérons alors la composée des morphisme naturels

$$
\operatorname{Pic}\left(V_{E}\right) \stackrel{\sim}{\longrightarrow} \operatorname{Pic}\left(V_{\operatorname{Fr}\left(\widehat{\mathscr{O}}_{\mathfrak{P}}^{\text {his }}\right)}\right) \stackrel{\sim}{\longrightarrow} \operatorname{Pic}\left(\mathscr{V}_{\widehat{\mathscr{O}_{\mathfrak{P}}^{\text {his }}}}\right) \rightarrow \operatorname{Pic}\left(\mathscr{V}_{\overline{\mathbf{F}}_{\mathfrak{P}}}\right)
$$

Par hypothèse, $H^{i}\left(V, \mathscr{O}_{V}\right)=0$ pour $i=1$ ou 2 . Par le théorème de semi-continuité (cf. [Ha theorem III.12.8]), on peut, quitte à augmenter $S$, supposer que $H^{i}\left(\mathscr{V}_{\mathbf{F}_{\mathfrak{p}}}, \mathscr{O}_{\mathscr{V}_{\mathbf{F}_{\mathfrak{p}}}}\right)$ est nul pour $i=1$ ou 2 et $\mathfrak{p} \in M_{F}-S$. Il en résulte que $H^{i}\left(\mathscr{V}_{\overline{\mathbf{F}}_{\mathfrak{p}}}, \mathscr{O}_{\mathscr{V}_{\bar{F}_{\mathfrak{p}}}}\right)$ est trivial pour $i=1$ ou 2. Par [Gr1] corollaire 1 de la proposition 3] la flèche de droite dans (2.2.1] est également un isomorphisme. On obtient ainsi l'isomorphisme recherché. 
En ce qui concerne la seconde assertion, le corang $\ell$-adique de $\operatorname{Br}\left(\mathscr{V}_{\overline{\mathbf{F}}_{\mathrm{p}}}\right)$ coïncide, d'après [Gr3, corollaire 3.4] avec la différence entre $B_{2}$, le deuxième nombre de Betti $\ell$-adique de $\mathscr{V}_{\overline{\mathbf{F}}_{\mathrm{p}}}$ et $\rho$ le rang du groupe de Picard de $\mathscr{V}_{\overline{\mathbf{F}}_{\mathrm{p}}}$. Par ce qui précède $\rho$ est également le rang de $\operatorname{Pic} \bar{V}$ et par [Se1, page 19-02] $B_{2}$ coïncide avec le deuxième nombre de Betti de $\bar{V}$. Par hypothèse la partie $\ell$-primaire de $\operatorname{Br}(\bar{V})$ est finie et $B_{2}=\rho$.

Convention 2.2.1. Dans la suite de ce texte, les paires $(S, \mathscr{V})$ considérées avec $S$ un ensemble fini de places de $F$ et $\mathscr{V}$ un modèle de $V$ sur $\mathscr{C}-S$ vérifient les conditions du lemme.

2.3. Mesures locales. Dans ce paragraphe, nous associons à toute métrique adélique (\| . $\left.\|_{\mathfrak{p}}\right)_{\mathfrak{p} \in M_{F}} \operatorname{sur} \omega_{V}^{-1}$ des mesures $\boldsymbol{\omega}_{\mathfrak{p}}$ sur $V\left(F_{\mathfrak{p}}\right)$.

Définition 2.3.1. Soit $\mathfrak{p}$ une place de $F$ et $\|\cdot\|_{\mathfrak{p}}$ une métrique $\mathfrak{p}$-adique sur $\omega_{V}^{-1}$. Si $x$ appartient à $V\left(F_{\mathfrak{p}}\right)$, on choisit des coordonnées locales $x_{1}, \ldots, x_{n}$ en $x$ définissant un morphisme de $F$ variétés $f$ d'un ouvert de Zariski $U$ de $V$ dans $\mathbf{A}_{F}^{n}$ et induisant un isomorphisme analytique pour la topologie p-adique d'un ouvert $W$ de $V\left(F_{\mathfrak{p}}\right)$ sur son image. Le morphisme de faisceau cohérent

$$
\omega(f): f^{*}\left(\omega_{\mathbf{A}_{F}^{n} / F}\right) \rightarrow \omega_{U / F}
$$

induit un isomorphisme de faisceau pour la topologie $\mathfrak{p}$-adique

$$
{ }^{t} \omega(f)^{-1}: f^{*} \omega_{f(W)}^{-1} \rightarrow \omega_{W}^{-1}
$$

qui permet d'identifier $\frac{\partial}{\partial x_{1}} \wedge \cdots \wedge \frac{\partial}{\partial x_{n}}$ avec une section de $\omega_{W}^{-1}$. Sur $W$, la mesure est alors définie par la relation

$$
\boldsymbol{\omega}_{\mathfrak{p}}=\left\|\frac{\partial}{\partial x_{1}} \wedge \cdots \wedge \frac{\partial}{\partial x_{n}}\right\|_{\mathfrak{p}} \mathrm{d} x_{1, \mathfrak{p}} \ldots \mathrm{d} x_{n, \mathfrak{p}}
$$

où $\mathrm{d} x_{i, \mathfrak{p}}$ désigne la mesures de Haar normalisée sur $F_{\mathfrak{p}}$.

Si $x_{1}, \ldots, x_{n}$ et $x_{1}^{\prime}, \ldots, x_{n}^{\prime}$ sont deux systèmes de coordonnées définies sur un même ouvert $W$ et correspondant à des fonctions

$$
f, f^{\prime}: U \rightarrow \mathbf{A}_{F}^{n}
$$

Soit $\Phi$ l'isomorphisme analytique

$$
f^{\prime} \circ f^{-1}: f(W) \rightarrow f^{\prime}(W)
$$

On a alors la relation

$$
{ }^{t} \omega(\Phi)^{-1}\left(\frac{\partial}{\partial x_{1}^{\prime}} \wedge \cdots \wedge \frac{\partial}{\partial x_{n}^{\prime}}\right)=\operatorname{Jac}_{x}(\Phi)^{-1} \frac{\partial}{\partial x_{1}} \wedge \cdots \wedge \frac{\partial}{\partial x_{n}}
$$

et par [We \$2.2.1], on a

$$
\mathrm{d} x_{1, \mathfrak{p}}^{\prime} \ldots \mathrm{d} x_{n, \mathfrak{p}}^{\prime}=\left|\operatorname{Jac}_{x}(\Phi)\right|_{\mathfrak{p}} \mathrm{d} x_{1, \mathfrak{p}} \ldots \mathrm{d} x_{n, \mathfrak{p}}
$$

On en déduit les égalités

$$
\begin{aligned}
& \left\|\frac{\partial}{\partial x_{1}^{\prime}} \wedge \cdots \wedge \frac{\partial}{\partial x_{n}^{\prime}}\right\|_{\mathfrak{p}} \mathrm{d} x_{1, \mathfrak{p}}^{\prime} \ldots \mathrm{d} x_{n, \mathfrak{p}}^{\prime} \\
& =\left\|\frac{\partial}{\partial x_{1}} \wedge \cdots \wedge \frac{\partial}{\partial x_{n}}\right\|_{\mathfrak{p}}\left|\operatorname{Jac}_{x}(\Phi)\right|_{\mathfrak{p}}^{-1} \mathrm{~d} x_{1, \mathfrak{p}}^{\prime} \ldots \mathrm{d} x_{n, \mathfrak{p}}^{\prime} \\
& =\left\|\frac{\partial}{\partial x_{1}} \wedge \cdots \wedge \frac{\partial}{\partial x_{n}}\right\|_{\mathfrak{p}} \mathrm{d} x_{1, \mathfrak{p}} \ldots \mathrm{d} x_{n, \mathfrak{p}} .
\end{aligned}
$$

Les mesures obtenues se recollent donc pour former une mesure borélienne $\omega_{\mathfrak{p}} \operatorname{sur} V\left(F_{\mathfrak{p}}\right)$. 
2.4. Lien avec les densités locales. Dans ce paragraphe, nous allons faire le lien pour $\mathfrak{p} \in M_{F}-S$ entre le volume $\boldsymbol{\omega}_{\mathfrak{p}}\left(V\left(F_{\mathfrak{p}}\right)\right)$ et la densité locale en $\mathfrak{p}$ définie par

$$
d_{\mathfrak{p}}(V)=\frac{\sharp \mathscr{V}\left(\mathbf{F}_{\mathfrak{p}}\right)}{\left(\sharp \mathbf{F}_{\mathfrak{p}}\right)^{\operatorname{dim} V}} .
$$

La démonstration suit celle de [We, §2.2.1], [Pe1] §2.2.2] et [Sa theorem 2.13].

Lemme 2.4.1. Pour presque toute place $\mathfrak{p}$ de $M_{F}-S$, on a :

$$
\omega_{\mathfrak{p}}\left(V\left(F_{\mathfrak{p}}\right)\right)=d_{\mathfrak{p}}(V) \text {. }
$$

Démonstration. Soit $n$ la dimension de $V$. Fixons un plongement $\Phi: V \rightarrow \mathbf{P}_{F}^{N}$ et $\mathscr{V}$ l'adhérence de $V$ dans $\mathbf{P}_{\mathscr{C}}^{N}$. Par le critère valuatif de propreté, on a une bijection de $V\left(F_{\mathfrak{p}}\right)$ $\operatorname{sur} \mathscr{V}\left(\mathscr{O}_{\mathfrak{p}}\right)$ qui induit pour tout $m$ des applications surjectives

$$
\pi_{m}: V\left(F_{\mathfrak{p}}\right) \rightarrow \mathscr{V}\left(\mathscr{O}_{\mathfrak{p}} / \mathfrak{p}^{m}\right)
$$

On note $[x]_{m}=\pi_{m}^{-1}\left(\pi_{m}(x)\right)$ pour tout $x$ de $V\left(F_{\mathfrak{p}}\right)$. Soit $I$ le faisceau d'idéaux défini par $V$ et $\mathscr{I}$ celui défini par $\mathscr{V}$. En dehors d'un nombre fini de places, le schéma $\mathscr{V}_{\mathscr{O}_{p}}$ est lisse et le faisceau $\left(\Lambda^{n} \Omega_{\mathscr{V}_{\mathscr{O}_{\mathfrak{p}}} / \mathscr{O}_{\mathfrak{p}}}\right)^{\vee}$ est un modèle de $\omega_{V}^{-1}$. On peut donc supposer que la métrique $\|\cdot\|_{\mathfrak{p}}$ est définie par ce modèle. Par [Ha theorem 8.1, theorem 8.13], on a des suites exactes de faisceaux

$$
\mathscr{I} / \mathscr{I}^{2} \stackrel{\delta}{\rightarrow} \Omega_{\mathbf{P}_{\mathscr{C}}^{N} / \mathscr{C}}^{1} \otimes \mathscr{O}_{\mathscr{V}} \stackrel{r}{\rightarrow} \Omega_{\mathscr{V} / \mathscr{C}}^{1} \rightarrow 0
$$

et

$$
0 \rightarrow J / J^{2} \stackrel{\delta}{\rightarrow} \Omega_{\mathbf{P}_{F}^{N} / F}^{1} \otimes \mathscr{O}_{V} \stackrel{r}{\rightarrow} \Omega_{V / F}^{1} \rightarrow 0 .
$$

Sur l'ouvert $\mathscr{V}_{i}$ défini par $X_{i} \neq 0$, on obtient des suites exactes

$$
\mathscr{I} / \mathscr{I}_{\mid \mathscr{V}_{i}}^{2} \stackrel{\delta}{\rightarrow} \bigoplus_{j \neq i} \mathscr{O}_{\mathscr{V}_{i}} \mathrm{~d} X_{j} \stackrel{r_{i}}{\rightarrow} \Omega_{\mathscr{V} / \mathscr{C}}^{1} \rightarrow 0
$$

et en notant $V_{i}=\mathscr{V}_{i F}$ qu'on identifie avec son image $U_{i}$ dans $\mathbf{A}_{F}^{N+1}$,

$$
J / J_{\mid U_{i}}^{2} \stackrel{\delta}{\rightarrow} \bigoplus_{j \neq i} \mathscr{O}_{U_{i}} \mathrm{~d} X_{j} \stackrel{r_{i}}{\rightarrow} \Omega_{U_{i} / F}^{1} \rightarrow 0
$$

Par conséquent pour presque toute place $\mathfrak{p}$ de $M_{F}$, on a

$$
\text { (2.4.3) } \forall x \in U_{i}\left(F_{\mathfrak{p}}\right), \quad \forall y \in \omega_{V}^{-1}(x), \quad\|y\|_{\mathfrak{p}} \underset{\substack{0 \leq i_{1}<\cdots<i_{n} \\ j \notin\left\{i_{1}, \ldots, i_{n}\right\}}}{=}\left|y\left(r_{i}\left(\mathrm{~d} X_{i_{1}}\right) \wedge \cdots \wedge r_{i}\left(d x_{i_{n}}\right)\right)\right|_{\mathfrak{p}} .
$$

Notons $f_{i}$ l'isomorphisme de $V_{i}$ sur $U_{i}$. On peut en outre supposer que $\mathfrak{p}$ vérifie les conditions I et II de [We page 19] pour la famille $\left(f_{i}\right)_{0 \leq i \leq N+1}$. Soit $x \in V\left(F_{\mathfrak{p}}\right)$ et $\left(x_{0}: \ldots: x_{N}\right)$ des coordonnées homogènes pour $\Phi(x)$. Après permutation des coordonnées et multiplication par un scalaire, on peut supposer que $x_{0}=1$ et $x_{1}, \ldots, x_{n} \in \mathscr{O}_{\mathfrak{p}}$. Le point $x$ provient alors d'un élément $\tilde{x}$ de $\mathscr{U}_{0}\left(\mathscr{O}_{\mathfrak{p}}\right)$. Par [We theorem 2.2.3], l'ensemble $[x]_{1}$ coïncide avec

$$
\left\{\left(1: x_{1}^{\prime}: \ldots: x_{n}^{\prime}\right) \in V\left(F_{\mathfrak{p}}\right) \mid x_{i}^{\prime} \in x_{i}+\mathfrak{p}\right\}
$$

On peut, à permutation des coordonnées près, supposer par 2.4.1) que $\Omega_{\mathscr{V}_{\mathscr{O}_{\mathfrak{p}}, x}^{1}}$ est isomorphe à $\bigoplus_{i=1}^{n} \mathscr{O}_{\mathscr{V}_{\mathscr{O}_{\mathfrak{p}}, x}} \mathrm{~d} X_{i}$. La famille $\left(X_{1}, \ldots, X_{n}\right)$ constitue alors un système de coordonnées locales au voisinage de $x$. Par [We page 22] ce système s'étend à $[x]_{1}$ et induit un difféomorphisme de $[x]_{1}$ sur $\left(x_{1}, \ldots, x_{n}\right)+\mathfrak{p}^{n}$ et l'isomorphisme entre $\Omega_{\mathscr{V}_{\mathscr{O}_{\mathfrak{p}}} / \mathscr{O}_{\mathfrak{p}}, x}^{1}$ et $\bigoplus_{i=1}^{n} \mathscr{O}_{\mathscr{V}_{\mathscr{O}_{\mathfrak{p}}}, x} \mathrm{~d} X_{i}$ s'étend également à $[x]_{1}$. Par conséquent 2 2.4.3) se réécrit alors

$$
\forall x^{\prime} \in[x]_{1}, \quad \forall y^{\prime} \in \omega_{V}^{-1}\left(x^{\prime}\right), \quad\left\|y^{\prime}\right\|_{\mathfrak{p}}=\left|\frac{y^{\prime}}{\frac{\partial}{\partial X_{1}} \wedge \cdots \wedge \frac{\partial}{\partial X_{n}}}\right|_{\mathfrak{p}}
$$


et sur $[x]_{1}$, la mesure $\omega_{\mathfrak{p}}$ coïncide avec

$$
\mathrm{d} X_{1, \mathfrak{p}} \ldots \mathrm{d} X_{n, \mathfrak{p}}
$$

On obtient donc

$$
\boldsymbol{\omega}_{\mathfrak{p}}\left([x]_{1}\right)=\int_{\left(x_{1}, \ldots, x_{n}\right)+\mathfrak{p}^{n}} \mathrm{~d} X_{1, \mathfrak{p}} \ldots \mathrm{d} X_{n, \mathfrak{p}}=\sharp \mathbf{F}_{\mathfrak{p}}^{-n} .
$$

En somman sur $\mathscr{V}\left(F_{\mathfrak{p}}\right)$, on obtient la formule du lemme.

2.5. Facteurs de convergence. Comme dans [Pe1] §2.2.3], nous allons maintenant appliquer les conjectures de Weil démontrées par Deligne pour obtenir des facteurs de convergence.

Définition 2.5.1. Pour tout $\mathfrak{p} \in M_{F}-S$, on note $\operatorname{Fr}_{\mathfrak{p}}$ le morphisme de Frobenius sur $\overline{\mathbf{F}}_{\mathfrak{p}}$ défini par $x \mapsto x^{\sharp \mathbf{F}_{\mathrm{p}}}$. La suite exacte

$$
0 \rightarrow I_{\mathfrak{p}} \rightarrow \operatorname{Gal}\left(\bar{F}_{\mathfrak{p}} / F_{\mathfrak{p}}\right) \rightarrow \operatorname{Gal}\left(\overline{\mathbf{F}}_{\mathfrak{p}} / \mathbf{F}_{\mathfrak{p}}\right) \rightarrow 0
$$

où $I_{\mathfrak{p}}$ désigne le groupe d'inertie en $\mathfrak{p}$ et l'inclusion canonique du groupe $\operatorname{Gal}\left(\bar{F}_{\mathfrak{p}} / F_{\mathfrak{p}}\right)$ dans $\operatorname{Gal}(\bar{F} / F)$ définissent une action de $\operatorname{Gal}\left(\overline{\mathbf{F}}_{\mathfrak{p}} / \mathbf{F}_{\mathfrak{p}}\right)$ sur $(\operatorname{Pic}(\bar{V}))^{I_{\mathfrak{p}}}$. On note également $\operatorname{Fr}_{\mathfrak{p}}$ le morphisme de Frobenius induit sur $(\operatorname{Pic}(\bar{V}))^{I_{\mathfrak{p}}}$. Le terme local de la fonction $L$ associée à $\operatorname{Pic}(\bar{V})$ est alors défini par

$$
L_{\mathfrak{p}}(s, \operatorname{Pic}(\bar{V}))=\frac{1}{\operatorname{Det}\left(1-\sharp \mathbf{F}_{\mathfrak{p}}^{-s} \operatorname{Fr}_{\mathfrak{p}} \mid(\operatorname{Pic}(\bar{V}))^{\left.I_{\mathfrak{p}} \otimes \mathbf{Q}\right)}\right.}
$$

Proposition 2.5.1. Pour toute place $\mathfrak{p}$ de $M_{F}-S$, le terme $L_{\mathfrak{p}}(1, \operatorname{Pic}(\bar{V}))$ est bien défini et le produit

$$
\prod_{\mathfrak{p} \in M_{F}} \frac{d_{\mathfrak{p}}(V)}{L_{\mathfrak{p}}(1, \operatorname{Pic}(\bar{V}))}
$$

est absolument convergent.

Démonstration. Par la démonstration du lemme 2.2.1 il existe une extension galoisienne finie $E$ de $F$ telle que $\operatorname{Pic}\left(V_{E}\right) \stackrel{\sim}{\longrightarrow} \operatorname{Pic}(\bar{V})$. L'action de $\operatorname{Gal}(\bar{F} / F)$ sur $\operatorname{Pic}(\bar{V})$ se factorise donc par $\operatorname{Gal}(E / F)$ et pour tout $\mathfrak{p} \in M_{F}$, on a que $\left(\operatorname{Fr}_{\mathfrak{p}}\right)^{[E: F]}$ agit trivialement sur $\operatorname{Pic}(\bar{V})^{I_{\mathfrak{p}}}$. Les valeurs propres de $\operatorname{Fr}_{\mathfrak{p}}$ sont donc des racines $[E: F]$-ièmes de l'unité et

$$
\operatorname{Det}\left(1-\sharp \mathbf{F}_{\mathfrak{p}}^{-s} \operatorname{Fr}_{\mathfrak{p}} \mid\left(\operatorname{Pic}(\bar{V})^{I_{\mathfrak{p}}} \otimes \mathbf{Q}\right)\right.
$$

est non nul ce qui montre la première assertion.

Par la formule de Lefschetz (cf. [Se2]) on a pour toute place $\mathfrak{p}$ en-dehors de $S$ et tout nombre premier $\ell$ distinct de $p$,

$$
\sharp \mathscr{V}\left(\mathbf{F}_{\mathfrak{p}}\right)=\sum_{i=0}^{2 n}(-1)^{i} \operatorname{Tr}\left(\operatorname{Fr}_{\mathfrak{p}} \mid H_{\text {ét }}^{i}\left(\mathscr{V}_{\overline{\mathbf{F}}_{\mathfrak{p}}}, \mathbf{Q}_{\ell}\right)\right)
$$

où $n$ désigne la dimension de $V$ et $\operatorname{Fr}_{\mathfrak{p}}$ le Frobenius géométrique. La variété $\mathscr{V}_{\overline{\mathbf{F}}_{\mathfrak{p}}}$ étant lisse, projective et géométriquement intègre, on a un isomorphisme canonique

$$
H_{\text {ét }}^{2 n}\left(\mathscr{V}_{\overline{\mathbf{F}}_{\mathfrak{p}}}, \mathbf{Q}_{\ell}(n)\right) \stackrel{\sim}{\longrightarrow} \mathbf{Q}_{\ell} .
$$

D'autre part les suites exactes de Kummer

$$
0 \rightarrow \mu_{\ell^{r}} \rightarrow \mathbf{G}_{m} \stackrel{\times \ell^{r}}{\rightarrow} \mathbf{G}_{m} \rightarrow 0
$$

où $r$ est positif induisent des suites exactes

et

$$
0 \rightarrow H_{\text {ét }}^{1}\left(\mathscr{V}_{\overline{\mathbf{F}}_{\mathfrak{p}}}, \mathbf{Z}_{\ell}(1)\right) \rightarrow T_{\ell}\left(\operatorname{Pic}\left(\mathscr{V}_{\overline{\mathbf{F}}_{\mathfrak{p}}}\right)\right) \rightarrow 0
$$

$$
0 \rightarrow \operatorname{Pic}\left(\mathscr{V}_{\overline{\mathbf{F}}_{\mathfrak{p}}}\right) \otimes \mathbf{Z}_{\ell} \rightarrow H_{\text {ét }}^{2}\left(\mathscr{V}_{\overline{\mathbf{F}}_{\mathfrak{p}}}, \mathbf{Z}_{\ell}(1)\right) \rightarrow T_{\ell}\left(\operatorname{Br}\left(\mathscr{V}_{\overline{\mathbf{F}}_{\mathfrak{p}}}\right)\right) \rightarrow 0
$$


Mais il résulte du lemme 2.2.1 que les modules de Tate

$$
T_{\ell}\left(\operatorname{Pic}\left(\mathscr{V}_{\overline{\mathbf{F}}_{\mathfrak{p}}}\right)\right)={\underset{n}{n}}_{\lim _{n}} \operatorname{Pic}\left(\mathscr{V}_{\overline{\mathbf{F}}_{\mathfrak{p}}}\right)_{\left(\ell^{n}\right)}
$$

et

$$
T_{\ell}\left(\operatorname{Br}\left(\mathscr{V}_{\overline{\mathbf{F}}_{\mathfrak{p}}}\right)\right)={\underset{\leftarrow}{n}}_{\lim _{n}} \operatorname{Br}\left(\mathscr{V}_{\overline{\mathbf{F}}_{\mathfrak{p}}}\right)_{\left(\ell^{n}\right)}
$$

sont tous les deux nuls. On obtient donc la trivialité du groupe $H_{\mathrm{ett}}^{1}\left(\mathscr{V}_{\overline{\mathbf{F}}_{\mathrm{p}}}, \mathbf{Q}_{\ell}(1)\right)$ et un isomorphisme

$$
H_{\mathrm{et}}^{2}\left(\mathscr{V}_{\overline{\mathbf{F}}_{\mathfrak{p}}}, \mathbf{Q}_{\ell}(1)\right) \stackrel{\sim}{\longrightarrow} \operatorname{Pic}\left(\mathscr{V}_{\overline{\mathbf{F}}_{\mathfrak{p}}}\right) \otimes \mathbf{Q}_{\ell}
$$

qui, par dualité de Poincaré, entraîne la trivialité de $H_{\mathrm{et}}^{2 n-1}\left(\mathscr{V}_{\overline{\mathbf{F}}_{\mathrm{p}}}, \mathbf{Q}_{\ell}\right)$ et induit un isomorphisme

$$
H_{\mathrm{et}}^{2 n-2}\left(\mathscr{V}_{\overline{\mathbf{F}}_{\mathrm{p}}}, \mathbf{Q}_{\ell}(n-1)\right) \stackrel{\sim}{\longrightarrow}\left(\operatorname{Pic}\left(\mathscr{V}_{\overline{\mathbf{F}}_{\mathrm{p}}}\right) \otimes \mathbf{Q}_{\ell}\right)^{\vee} .
$$

Or pour toute paire d'eniers $i$ et $j$, on a

$$
\operatorname{Tr}\left(\operatorname{Fr}_{\mathfrak{p}} \mid H_{\mathrm{et}}^{i}\left(\mathscr{V}_{\overline{\mathbf{F}}_{\mathfrak{P}}}, \mathbf{Q}_{\ell}(j)\right)=\frac{1}{\sharp \mathbf{F}_{\mathfrak{p}}^{j}} \operatorname{Tr}\left(\operatorname{Fr}_{\mathfrak{p}} \mid H_{\mathrm{et}}^{i}\left(\mathscr{V}_{\overline{\mathbf{F}}_{\mathfrak{p}}}, \mathbf{Q}_{\ell}\right) .\right.\right.
$$

Par conséquent

$$
d_{\mathfrak{p}}(V)=1+\frac{1}{\sharp \mathbf{F}_{\mathfrak{p}}} \operatorname{Tr}\left(\operatorname{Fr}_{\mathfrak{p}} \mid \operatorname{Pic}\left(\mathscr{V}_{\overline{\mathbf{F}}_{\mathfrak{p}}}\right) \otimes \mathbf{Q}_{\ell}\right)+\sum_{i=0}^{2 n-3} \frac{(-1)^{i}}{\sharp \mathbf{F}_{\mathfrak{p}}^{\operatorname{dim} V} V} \operatorname{Tr}\left(\operatorname{Fr}_{\mathfrak{p}} \mid H_{\mathfrak{e t}}^{i}\left(\mathscr{V}_{\overline{\mathbf{F}}_{\mathfrak{p}}}, \mathbf{Q}_{\ell}\right)\right) .
$$

Mais par la conjecture de Weil démontrée par Deligne sur les valeurs propres des endomorphismes de Frobenius [Del] theorem 1.6], on a des inégalités

$$
\left|\operatorname{Tr}\left(\operatorname{Fr}_{\mathfrak{p}} \mid H_{\mathrm{ett}}^{i}\left(\mathscr{V}_{\overline{\mathbf{F}}_{\mathfrak{p}}}, \mathbf{Q}_{\ell}\right)\right)\right| \leq \sharp \mathbf{F}_{\mathfrak{p}}^{i / 2} \operatorname{dim} H_{\mathrm{ett}}^{i}\left(\mathscr{V}_{\overline{\mathbf{F}}_{\mathfrak{p}}}, \mathbf{Q}_{\ell}\right) .
$$

Or le $i$-ième nombre de Betti étale $\operatorname{dim} H_{\mathrm{ett}}^{i}\left(\mathscr{V}_{\overline{\mathbf{F}}_{\mathfrak{p}}}, \mathbf{Q}_{\ell}\right)$ est constant sur les places $\mathfrak{p}$ de bonne réduction (cf. [Se1] page 19-02]) et donc

$$
d_{\mathfrak{p}}(V)=1+\frac{1}{\sharp \mathbf{F}_{\mathfrak{p}}} \operatorname{Tr}\left(\operatorname{Fr}_{\mathfrak{p}} \mid \operatorname{Pic}\left(\mathscr{V}_{\overline{\mathbf{F}}_{\mathfrak{p}}}\right) \otimes \mathbf{Q}\right)+O\left(\frac{1}{\sharp \mathbf{F}_{\mathfrak{p}}^{3 / 2}}\right)
$$

D'autre part les valeurs propres de $\operatorname{Fr}_{\mathfrak{p}} \operatorname{sur} \operatorname{Pic}\left(\mathscr{V}_{\overline{\mathbf{F}}_{\mathfrak{p}}}\right)$ qui est isomorphe à $\operatorname{Pic}\left(V_{E}\right)$ étant des racines de l'unité, on a également

$$
\operatorname{Det}\left(1_{\sharp} \mathbf{F}_{\mathfrak{p}}^{-1} \operatorname{Fr}_{\mathfrak{p}} \mid \operatorname{Pic}\left(\mathscr{V}_{\overline{\mathbf{F}}_{\mathfrak{p}}}\right) \otimes \mathbf{Q}\right)=1+\frac{1}{\sharp \mathbf{F}_{\mathfrak{p}}} \operatorname{Tr}\left(\operatorname{Fr}_{\mathfrak{p}} \mid \operatorname{Pic}\left(\mathscr{V}_{\overline{\mathbf{F}}_{\mathfrak{p}}}\right) \otimes \mathbf{Q}\right)+O\left(\frac{1}{\sharp \mathbf{F}_{\mathfrak{p}}^{3 / 2}}\right)
$$

et il en résulte que

$$
\frac{d_{\mathfrak{p}}(V)}{L \mathfrak{p}(1, \operatorname{Pic}(\bar{V}))}=1+O\left(\frac{1}{\sharp \mathbf{F}_{\mathfrak{p}}^{3 / 2}}\right.
$$

et le produit de ces termes converge absolument.

Définition 2.5.2. Pour toute place $\mathfrak{p}$ de $F$, on pose

$$
\lambda_{\mathfrak{p}}=L_{\mathfrak{p}}(1, \operatorname{Pic}(\bar{V})) .
$$


2.6. Mesure de Tamagawa. Afin de normaliser le mesure nous aurons besoin de prendre le résidu de la fonction $L$ associée à $\operatorname{Pic} \bar{V}$ et donc du lemme suivant :

Lemme 2.6.1. Le produit eulérien

$$
\prod_{\mathfrak{p} \in M_{F}} L_{\mathfrak{p}}(s, \operatorname{Pic}(\bar{V}))
$$

converge absolument pour $\operatorname{Re} s>1$ et définit une fonction $L(s, \operatorname{Pic}(\bar{V}))$ qui se prolonge en une fonction rationnelle de $q^{-s}$ sur $\mathbf{C}$ avec un pôle d'ordre $t=\operatorname{rg} \operatorname{Pic}(V)$ en $s=1$.

Démonstration. La convergence pour $\operatorname{Re} s>1$ résulte de la définition et du fait que les valeurs propres du Frobenius agissant $\operatorname{sur} \operatorname{Pic}(\bar{V})$ sont des racines de l'unité.

Soit $\operatorname{Fr}_{q_{F}}$ l'élément du groupe $\operatorname{Gal}(\bar{F} / F)$ envoyant $x$ sur $x^{q_{F}}$. La fonction $L$ est alors donnée par le produit eulérien

$$
L\left(s, \operatorname{Pic}(\bar{V})=\prod_{x \in \mathscr{C}(0)} \frac{1}{\operatorname{Det}\left(1-\sharp \kappa(x)^{-s} \operatorname{Fr}_{q_{F}}^{\left[\kappa(x): \mathbf{F}_{q_{F}}\right]} \mid \operatorname{Pic}(\bar{V}) \otimes \mathbf{Q}\right)}\right.
$$

où $\mathscr{C}_{(0)}$ désigne l'ensemble des points fermés de $\mathscr{C}$ et $\kappa(x)$ le corps résiduel en $x$. Notons $\left(\alpha_{i}\right)_{i \in I}$ les valeurs propres de $\operatorname{Fr}_{q_{F}}$ agissant $\operatorname{sur} \operatorname{Pic}(\bar{V}) \otimes \mathbf{Q}$ et $\left(m_{i}\right)_{i \in I}$ leurs multiplicités. On obtient

$$
\begin{aligned}
L(s, \operatorname{Pic}(\bar{V})) & =\prod_{i \in I} \prod_{x \in \mathscr{C}_{(0)}} \frac{1}{\left(1-\left(q^{-s} \alpha_{i}\right)^{\left[\kappa(x): \mathbf{F}_{q_{F}}\right]}\right)^{m_{i}}} \\
& =\prod_{i \in I} Z\left(\mathscr{C}, q^{-s} \alpha_{i}\right)
\end{aligned}
$$

où $Z(\mathscr{C}, t)$ est la fonction zêta de $\mathscr{C}$ définie par

$$
Z(\mathscr{C}, t)=\exp \left(\sum_{n>0} \frac{\sharp \mathscr{C}\left(\mathbf{F}_{q_{F}^{n}}\right) t^{n}}{n}\right) .
$$

Par le théorème de Weil $Z(\mathscr{C}, t)$ est une fonction rationnelle de $t$ avec un pôle d'odre 1 en $t=q^{-1}$. De manière plus précise, on a en fait

$$
Z(\mathscr{C}, t)=\frac{\operatorname{Det}\left(1-t \mathrm{Fr}_{q_{F}} \mid H_{\mathrm{et}}^{1}\left(\mathscr{C}_{\overline{\mathbf{F}}_{q_{F}}}\right)\right)}{(1-t)(1-q t)}
$$

Ceci implique la deuxième assertion.

Définition 2.6.1. On considère la mesure de Tamagawa

$$
\boldsymbol{\omega}_{H}=\left(\lim _{s \rightarrow 1}(s-1)^{t} L(s, \operatorname{Pic}(\bar{V}))\right) \frac{1}{q_{F}^{\left(g_{F}-1\right) \operatorname{dim} V}} \prod_{\mathfrak{p} \in M_{F}} \lambda_{\mathfrak{p}}^{-1} \boldsymbol{\omega}_{\mathfrak{p}}
$$

et le nombre de Tamagawa de $V$ relativement à la hauteur $H$ est défini par

$$
\tau_{H}(V)=\boldsymbol{\omega}_{H}(\overline{V(F)})
$$

où $\overline{V(F)}$ désigne l'adhérece des points rationnels de $V$ dans l'espace adélique $V\left(\boldsymbol{A}_{F}\right)$.

\section{Présentation du Résultat}

3.1. Facteurs $\alpha(V)$ et $\beta(V)$. Nous allons maintenant définir l'analogue de la fonction caractéristique $\chi_{C_{\text {eff }}(V)}$, qui remonte à [Kö], et qui fut introduite dans le contexte des conjectures de Manin par Batyrev et Tschinkel dans [BT1]. Cette analogue est fourni par le facteur local de la fonction $L$ de Draxl associée au cône effectif (cf. [Dr]). 
Définition 3.1.1. Soit $M$ un Z-module libre et $C$ un cône rationnel polyédrique strictement convexe de $M \otimes \mathbf{R}$; c'est-à-dire qu'il existe une famille finie $\left(m_{i}\right)_{1 \leqslant i \leqslant r}$ d'éléments de $M$ telle que $C=\sum_{i=1}^{r} \mathbf{R}_{\geq 0} m_{i}$ avec $C \cap-C=\{0\}$. On note $C^{\vee}$ le cône dual défini par

$$
C^{\vee}=\left\{y \in(M \otimes \mathbf{R})^{\vee} \mid \forall x \in C,\langle x, y\rangle \geqslant 0\right\}
$$

et on pose pour tout $s \in \stackrel{\circ}{C}+i M$,

$$
L_{q}(s, M, C)=\sum_{y \in C^{\vee} \cap M^{\vee}} q^{-\langle y, s\rangle} .
$$

Par définition du cône dual cette série converge absolument sur le cône ouvert $\stackrel{\circ}{C}+i M$ et si $m$ appartient à l'intérieur de $C$, la fonction qui à $s$ associe $L_{q}(s m, M, C)$ a un pôle d'ordre $\operatorname{rg} M$ en $s=0$.

On pose

$$
\alpha^{*}(V)=(\log q)^{t} \lim _{s \rightarrow 1}(s-1)^{t} L_{q}\left(s \omega_{V}^{-1}, \operatorname{Pic}(V), C_{\text {eff }}(V)\right)
$$

où $t$ désigne le rang de $\operatorname{Pic}(V)$ et

$$
\alpha(V)=\frac{\alpha^{*}(V)}{(t-1) !}
$$

Enfin, comme Batyrev et Tschinkel, on pose

$$
\beta(V)=\sharp H^{1}(F, \operatorname{Pic}(\bar{V}))
$$

bien que ce terme soit trivial dans les cas considérés ici.

Remarque 3.1.1. La fonction $L_{q}(s, M, C)$ est périodique de groupe de période contenant $(2 \pi i / \log q) M$. Il résulte de la définition que $\alpha^{*}(V)$ dépend du réseau $\operatorname{Pic}(V)$, du cône $C_{\text {eff }}(V)$ et de l'élément $\omega_{V}^{-1}$ mais est indépendant de $q$.

Notation 3.1.2. La fonction caractéristique du cône $C_{\text {eff }}(V)$ est définie par

$$
\forall s \in \overparen{C_{\text {eff }}(V)}, \quad \chi_{C_{\text {eff }}(V)}(s)=\int_{C_{\text {eff }}(V) \vee} e^{-\langle s, y\rangle} \mathrm{d} y .
$$

Définition 3.1.3. Si $f$ est une fonction méromorphe sur un ouvert d'un C-espace vectoriel $W$, nous dirons que $f$ admet une expression rationnelle en des puissances de $q$ s'il existe une base $\left(\chi_{i}\right)_{1 \leq i \leq n}$ de $W^{\vee}$ et une fonction rationnelle

$$
R \in \mathbf{C}\left(T_{1}, \ldots, T_{n}\right)
$$

telle que pour tout $s$ en lequel $f$ est défini on ait

$$
f(s)=R\left(q^{\left\langle\chi_{1}, s\right\rangle}, \ldots, q^{\left\langle\chi_{n}, s\right\rangle}\right) .
$$

Proposition 3.1.2. Avec les hypothèses précédentes, la fonction $L_{q}\left(\cdot, \operatorname{Pic}(V), C_{\mathrm{eff}}(V)\right)$ admet une expression rationnelle en des puissances de q et

$$
\alpha^{*}(V)=\chi_{C_{\text {eff }}(V)}\left(\omega_{V}^{-1}\right) \in \mathbf{Q}^{*} .
$$

Remarque 3.1.3. La raison pour laquelle nous avons substitué $L_{q}$ à $\chi_{C_{\text {eff }}(V)}$ dans ce cadre est, qu'étant péridique comme la fonction zêta des hauteurs lorsque le système de hauteurs vérifie la propriété $(\mathrm{P}), L_{q}\left(\cdot, \operatorname{Pic}(V), C_{\text {eff }}(V)\right)$ devrait avoir même lieu singulier que $\zeta_{U, \boldsymbol{H}}$ au voisinage de $\omega_{V}^{-1}+i \operatorname{Pic}(V) \otimes \mathbf{R}$. 
Démonstration. Rappelons qu'un cône $C$ de $M \otimes \mathbf{R}$ est dit régulier s'il est de la forme

$$
\sum_{i=1}^{r} m_{i}
$$

où $\left(m_{i}\right)_{1 \leqslant i \leqslant r}$ est une famille libre de $M$. Plaçons-nous tout d'abord dans le cas où $C$ est une cône régulier d'intérieur non vide. La fonction $L_{q}(\cdot, M, C)$ s'écrit alors

$$
L_{q}(s, M, C)=\prod_{i=1}^{n}\left(1-q^{\left\langle m_{i}, s\right\rangle}\right)^{-1}
$$

et la première assertion est immédiate. En outre, si $s=\sum_{i=1}^{n} s_{i} m_{i}$ alors par [BT1] proposition 2.4.5], on a l'égelité

$$
\chi_{C}(s)=\prod_{i=1}^{n} \frac{1}{s_{i}}
$$

la deuxième assertion en découle aussitôt. Dans le cas général (cf. [Oda p. 23]), on écrit $C^{\vee}$ comme support d'un éventail régulier $\Sigma$, c'est-à-dire que $\Sigma$ est un ensemble de cônes polyédriques rationnels strictement convexes de $M^{\vee} \otimes \mathbf{R}$ tels que :

(i) si $\sigma \in \Sigma$ et si $\sigma^{\prime}$ est une face de $\sigma$, alors $\sigma^{\prime} \in \Sigma$,

(ii) si $\sigma, \sigma^{\prime} \in \Sigma$, alors $\sigma \cap \sigma^{\prime}$ est une face de $\sigma$ et de $\sigma^{\prime}$.

(iii) $C^{\vee}=\cup_{\sigma \in \Sigma} \sigma$,

(iv) tout $\sigma$ de $\Sigma$ est régulier.

Alors si on note $\Sigma^{(i)}$ l'ensemble des éléments de $\Sigma$ de dimension $i$ et $n=\operatorname{dim} M$, on a les égalités :

$$
L_{q}(s, M, C)=\sum_{\sigma \in \Sigma^{(n)}} L_{q}\left(s, M, \sigma^{\vee}\right)
$$

et

$$
\chi_{C}(s)=\sum_{\sigma \in \Sigma^{(n)}} \chi_{C}(s)
$$

et les deux assertions résultent du cas précédent.

3.2. Expression de la constante. Nous allons maintenant définir la constante qui apparaît comme résidu de la fonction zêta des hauteurs.

Définition 3.2.1. On pose

$$
\theta_{H}^{*}(V)=\alpha^{*}(V) \beta(V) \tau_{H}(V)
$$

et

$$
\theta_{H}(V)=\alpha(V) \beta(V) \tau_{H}(V)
$$

3.3. Géométrie des variétés de drapeaux généralisées. Dans la suite nous nous intéressons à la situation suivante :

Notations 3.3.1. Dans la suite $G$ désigne un groupe algébrique linéaire lisse semi-simple et connexe sur $F, P$ un $F$-sous-groupe parabolique lisse de $G$. On note $V$ le quotient $P \backslash G$ et $\pi: G \rightarrow V$ la projection canonique. Par [BoTi2 proposition 2.24], le revêtement universel $\tilde{G}$ de $G$ est défini sur $F$. Quitte a remplacer $G$ par $\tilde{G}$ et $P$ par son image inverse dans $\tilde{G}$, on peut donc supposer que $G$ est simplement connexe.

Pour tout groupe algébrique linéaire $H$ sur $F$, on note Lie $(H)$ l'algèbre de Lie restreinte de $H, \mathscr{R} H$ son radical et $\mathscr{R}_{u} H$ son radical unipotent. Le groupe des caractères de $H$ sur $F$ est défini par :

$$
X^{*}(H)_{F}=\operatorname{Hom}_{\operatorname{Spec} F}\left(H, \mathbf{G}_{m, F}\right)
$$

et le groupe de cocaractères par

$$
X_{*}(H)_{F}=\operatorname{Hom}_{\operatorname{Spec} F}\left(\mathbf{G}_{m, F}, H\right) .
$$


On note $P_{0}$ un $F$-sous-groupe parabolique lisse minimal de $G$ contenu dans $P$. On note $T$ un tore maximal de $\mathscr{R} 0$ et $S$ sa composante scindée. On a donc les inclusions

$$
S \subset T \subset P_{0} \subset P \subset P \subset G \text {. }
$$

On fixe également un sous-groupe de Borel $B$ de $G^{s}$ tel que $T^{s} \subset B \subset P_{0}^{s}$.

On note $\Phi$ (resp. ${ }_{F} \Phi, \Phi^{+},{ }_{F} \Phi^{+}$) les racines de $T^{s}$ (resp. $S, T^{s}, S$ ) dans $G^{s}$ (resp. $G, B$, $\left.P_{0}\right), \Delta$ le base de $\Phi$ associée à $\Phi^{+}$et ${ }_{F} \Delta$ celle de ${ }_{F} \Phi$ correspondant à ${ }_{F} \Phi^{+}$. L'application de restriction de $T$ à $S$ induit une application

$$
j: \Delta \rightarrow{ }_{F} \Delta \cup\{0\}
$$

(cf. [Bo §21.8]) dont l'image contient ${ }_{F} \Delta$. le groupe de Weyl de $\Phi$ (resp. ${ }_{F} \Phi$ ) est note $W$ (resp. ${ }_{F} W$ ). Pour tout $\alpha$ de $\Delta$ (resp. ${ }_{F} \Delta$ ), on note $\check{\alpha}$ la coracine correspondante et $\varpi_{\alpha}$ le poids fondamental associé.

Pour tout partie $J$ de ${ }_{F} \Delta$, on note ${ }_{F} W_{J}$ le sous-groupe de ${ }_{F} W$ engendrépar les $s_{\alpha}$, pour $\alpha \in J$ et ${ }_{F} P_{J}$ le $F$ sous-groupe parabolique

$$
{ }_{F} P_{J}=P_{0 F} W_{J} P_{0} .
$$

On obtient ainsi une bijection entre les parties de ${ }_{F} \Delta$ et les $F$ sous-groupes paraboliques de $G$ contenant $P_{0}$ avec

$$
P_{0}={ }_{F} P_{\emptyset} \quad \text { et } \quad G={ }_{F} P_{F} \Delta .
$$

On note ${ }_{F} I$ (resp. I) la partie de ${ }_{F} \Delta$ (resp. $\Delta$ ) correspondant à $P$. On a donc

$$
I=j^{-1}\left({ }_{F} I \cup\{0\}\right)
$$

Par [San proposition 6.10], on a une suite exacte

$$
0 \rightarrow \mathbf{F}[V]^{*} / F^{*} \rightarrow F[G]^{*} / F^{*} \rightarrow X^{*}(P)_{F} \rightarrow \operatorname{Pic}(V) \rightarrow \operatorname{Pic}(G)
$$

Il résulte du théorème de Rosenlicht, [Ro theorem 3] que $F[G]^{*} / F^{*}$ est isomorphe au groupe $X^{*}(G)_{F}$ et donc trivial et de $[\operatorname{San}$ lemma 6.9 (iii)] que $\operatorname{Pic}(G)$ est nul puisque $G$ est supposé simplement connexe. On a donc un isomorphisme canonique

$$
\phi: X^{*}(P)_{F} \stackrel{\sim}{\longrightarrow} \operatorname{Pic}(V)
$$

qui peut être décrit de la maniére suivante : si $\chi$ est un caractère de $P$ sur $F, \chi$ peut être vu comme fibré en droites sur $\operatorname{Spec} F$ muni d'une action de $P$ et comme fibré en droites sur $V, \phi(\chi)$ est défini comme le produit restreint $G \times{ }^{P} \chi$. Autrement dit $\phi(\chi)$ est la classe du faisceau $L_{\chi}$ défini par

$\Gamma\left(U, L_{\chi}\right)=\left\{f \in \Gamma\left(\pi^{-1}(U), \mathscr{O}_{G}\right) \mid \forall y \in \pi^{-1}(U)(\bar{F}), \forall p \in P(\bar{F}), f(p g)=\chi(p) f(g)\right\}$

Par [Pe1, lemme 6.2.10], l'image de $X^{*}(P)_{F^{s}}$ dans $X^{*}(T)_{F^{s}}$ coïncide avec le sous réseau engendré par la famille $\left(\varpi_{\alpha}\right)_{\alpha \in \Delta-I}$ et le cône des diviseurs effectifs est donné par

$$
C_{\text {eff }}\left(V^{s}\right)=\sum_{\alpha \in \Delta-I} \mathbf{R}_{\geq 0}\left(-\varpi_{\alpha}\right) .
$$

L'image de $X^{*}(P)_{F}$ dans $X^{*}(T)_{F^{s}}$ a donc pour base la famille

$$
\left(\sum_{\beta \in j^{-1}(\alpha)} \varpi_{\beta}\right)_{\alpha \in{ }_{F} \Delta-{ }_{F} I}
$$

et $C_{\text {eff }}(V)$ est donné par

$$
\sum_{\alpha \in{ }_{F} \Delta-{ }_{F} I} \mathbf{R}_{\geq 0}\left(-\sum_{\beta \in j^{-1}(\alpha)} \varpi_{\beta}\right) .
$$


Pour tout $J \subset{ }_{F} \Delta$, on note ${ }_{F} \mathfrak{r}_{J}$ le radical de $\operatorname{Lie}\left({ }_{F} P_{J}\right)$ et on pose $\mathfrak{r}={ }_{F} \mathfrak{r}_{F}{ }$. La représentation adjointe définit une action de $P$ sur $\mathfrak{r}$ et le fibré cotangent $\Omega_{V / F}^{1}$ est isomorphe au fibré $G \times{ }^{P} \mathfrak{r}$ associé. En prennt les puissances extérieures maximales, on obtient que

On note

$$
\phi(\operatorname{det} \mathfrak{r})=\omega_{V}
$$

$$
\rho_{P}=\frac{1}{2} \operatorname{det} \mathfrak{r} \in X^{*}(P)_{F} \otimes \mathbf{Q}
$$

L'image de $\rho_{P}$ dans $X^{*}(S)_{F}$ par la restriction coïncide avec la demi-somme des racines de $\mathrm{S}$ comptées avec des multiplicitées égales à la dimension de leur espace propre dans $\mathfrak{r}$.

Notons en outre que tout choix de bases des sous-espaces propres de ${ }_{F} \mathfrak{r}_{\emptyset}$ pour l'action de $S$ définit un isomorphisme de $F$-espace vectoriel det $\mathfrak{r} \stackrel{\sim}{\longrightarrow} F$ et donc un isomorphisme

$$
\omega_{V} \stackrel{\sim}{\longrightarrow} L_{2 \rho_{P}} \text {. }
$$

Remarque 3.3.1. Il résulte des descriptions de $C_{\text {eff }}(V)$ et de $\omega_{V}^{-1}$ que $\omega_{V}^{-1} \in \overparen{C_{\text {eff }}(V)}$ et $V$ vérifie l'hypothèse (i) des hypothèses géométriques ainsi qu l'hypothèse (iv). L'hypothèse (ii) résulte de [Ke p. 575] et, du fait que $P$ est supposé réduit, la condition (iii) résulte de la description du groupe du Picard et la dernière du fait que $\bar{V}$ est rationnelle. La variété vérifie donc l'ensemble des hypothèses géométriques.

3.4. Hauteurs sur les variétés de drapeaux. Comme dans [FMT], nous allons tout d'abord nous restreindre au cas des hauteurs définies par des sous-groupes compacts maximaux. Le but de ca paragraphe est d'en rappeler la construction.

Notations 3.4.1. Par la décomposition d'iwasawa (cf. [Tit \$3.3.2]), Il existe pour toute place $\mathfrak{p}$ de $F$ un sous-groupe compact maximal $K_{\mathfrak{p}}$ de $G\left(F_{\mathfrak{p}}\right)$ tel que

$$
G\left(F_{\mathfrak{p}}\right)=P_{0}\left(F_{\mathfrak{p}}\right) K_{\mathfrak{p}}
$$

En outre si $\mathscr{G}$ est un modèle de $G$ sur un ouvert de $\mathbf{C}$, alors par [Tit §3.9.1], on peut supposer pour presque toute place $\mathfrak{p}$ de $F$ que

$$
K_{\mathfrak{p}}=\mathscr{G}\left(\mathscr{O}_{\mathfrak{p}}\right)
$$

On pose $K=\prod_{\mathfrak{p} \in M_{F}} K_{\mathfrak{p}}$. Pour tout caractère $\chi$ de $P$, pour toute place $\mathfrak{p}$ de $F$, on considère la métrique $\|\cdot\|_{\mathfrak{p}}$ sur $L_{\chi}$ définie de la manière suivante : si $U$ est un voisinage ouvert de $x, s$ une section de $L_{\chi}$ non nulle en $x$ et $\widetilde{s}$ l'élément de $\Gamma\left(\pi^{-1}(U), \mathscr{O}_{G}\right)$ qui lui correspond,

$$
\forall k \in K_{\mathfrak{p}}, \quad \pi(k)=x \Rightarrow\|s(x)\|_{\mathfrak{p}}=|\widetilde{s}(k)|_{\mathfrak{p}} .
$$

L'existence d'un tel $k$ est assuré par (3.4.1), et le terme de droite est in dépendant de $k$ puisque pour tout morphisme continu de $P\left(F_{\mathfrak{p}}\right) \cap K_{\mathfrak{p}}$ dans $\mathbf{R}_{>0}$ est trivial.

Le système de métriques $\left(\|\cdot\|_{\mathfrak{p}}\right)_{\mathfrak{p} \in M_{F}}$ ainsi défini est bien adélique. En effet soit $\mathscr{P}$ l'adhérence de $P$ dans $\mathscr{G}$. Quitte à augmenter $S$, on peut supposer que $\mathscr{P}$ est un sousgroupe parabolique de $\mathscr{G}$ sur $\mathbf{C}-S$ et le quotient $\mathscr{P} \backslash \mathscr{G}$, bien défini par [Dem proposition 1.2], fournit un modèle $\mathscr{V}$ de $V$ sur $\mathbf{C}-S$. On considère alors le faisceau $\mathscr{L}_{\chi}$ sur $\mathscr{V}$ défini par

$\Gamma\left(\mathscr{U}, \mathscr{L}_{\chi}\right)=\left\{f \in \Gamma\left(\pi^{-1}\left(\mathscr{U}, \mathscr{O}_{\mathscr{G}}\right) \mid \forall g \in \pi^{-1}(\mathscr{U})(\bar{F}), \forall p \in P(\bar{F}), f(p g)=\chi(p) f(g)\right\}\right.$ pour tout ouvert $\mathscr{U}$ de $\mathscr{V}$. Quitte à augmenter $S$, on a que $\mathscr{L}_{\chi}$ est un fibré en droites et un modèle de $L_{\chi}$ et pour tout $\mathfrak{p} \in M_{F}-S$ tel que $K_{\mathfrak{p}}=\mathscr{G}\left(\mathscr{O}_{\mathfrak{p}}\right)$ et tout $x$ de $V\left(F_{\mathfrak{p}}\right)$ se relevant en un élément $k$ de $K_{\mathfrak{p}}$, la $\mathscr{O}_{\mathfrak{p}}$-structure de $L_{\chi}(x)$ définie par $L_{\chi}$ est induite par la $\mathscr{O}_{\mathfrak{p}}$-structure de $\mathscr{O}_{G}(c)$ induite par $\mathscr{O}_{\mathscr{G}}$, ce qui mongtre que $\|\cdot\|_{\mathfrak{p}}$ coïncide avec la métrique définie par $\mathscr{L}_{\chi}$.

L'application

$$
\chi \mapsto\left(L_{\chi},\left(\|\cdot\|_{\mathfrak{p}}\right)_{\mathfrak{p} \in M_{F}}\right)
$$


définit un système de métriques adéliques sur $V$ qui vérifie de surcroît la propiété $(\mathrm{P})$. Nous noterons $\boldsymbol{H}_{K}$ ce système de hauteurs. Nous omettrons $K$ dans cette notation lorsqu' aucune confusion ne sera possible.

3.5. Enoncé du résultat. Nous pouvons maintenant énocer notre résultat proncipal :

Théorème 3.5.1. Avec les notations qui précèdent, la fonction zêta des hauteurs $\zeta_{V, \boldsymbol{H}_{K}}(s)$ converge absolument pour

$$
s \in \omega_{V}^{-1}+\overbrace{C_{\text {eff }}(V)}^{\circ}+i \operatorname{Pic} V \otimes \mathbf{R}
$$

et sétend à $\mathrm{Pic} V \otimes \mathbf{C}$ en une fonction méromorphe qui admet une expression rationnelle en des puissance de q. En outre la fonction méromorphe sur $\mathbf{C}$ qui à s associe $\zeta_{V, H_{K}}\left(s \omega_{V}^{-1}\right)$ a un pôle d'ordre $t=\operatorname{rg} \operatorname{Pic} V$ en $s=1$ avec

$$
\lim _{s \rightarrow 1}(s-1)^{t} \zeta_{V, \boldsymbol{H}_{K}}\left(s \omega_{V}^{-1}\right)=\theta^{*}(V) .
$$

\section{DÉmonstration DU RÉSUltat}

4.1. Fonction zêta et séries d'Eisenstein. Comme dans [FMT] §2], la démonstration est basée sur le fait que la série zêta des hauteurs coïncide avec une série d'Eisenstein, ce qui permet d'appliquer les résultats démontrés par Morris dans [Mo1] et [Mo2] pour ces séries.

Notations 4.1.1. Pour toute partie $J$ de ${ }_{F} \Delta$, on note $S_{J}$ le tore

$$
\left(\bigcap_{\alpha \in J} \operatorname{Ker} \alpha\right)^{\circ}
$$

où pour tout groupe algébrique $H$, on désigne par $H^{\circ}$ sa composante neutre. On pose également

$$
{ }_{F} M_{J}=Z_{G}\left(S_{J}\right) \quad \text { et } \quad{ }_{F} N_{J}=\mathscr{R}_{u}\left({ }_{F} P_{J}\right) .
$$

Le groupe parabolique ${ }_{F} P_{J}$ est alors le produit semi-direct de ${ }_{F} M_{J}$ par ${ }_{F} N_{J}$. La restriction iduit un isomorphisme

$$
X^{*}\left({ }_{F} P_{J}\right) \stackrel{\sim}{\longrightarrow} X^{*}\left({ }_{F} M_{J}\right)
$$

Dans la suite, on posera

$$
\mathfrak{a}_{J}=X^{*}\left({ }_{F} M_{J}\right) \otimes_{\mathbf{z}} \mathbf{C} \stackrel{\stackrel{\text { Res }}{\sim}}{\longrightarrow} X^{*}\left(S_{J}\right) \otimes_{\mathbf{Z}} \mathbf{C}
$$

et on notera $M$ (resp. $N$, a $, M_{0}, N_{0}, \mathfrak{a}_{0}$ ) pour ${ }_{F} M_{F} I$ (resp. ${ }_{F} N_{F}, \mathfrak{a}_{F} I,{ }_{F} M_{\emptyset},{ }_{F} N_{\emptyset}$, $\left.\mathfrak{a}_{\emptyset}\right)$.

Pour tout place $\mathfrak{p}$ de $F$, on définit un morphisme

$$
H_{P, \mathfrak{p}}: M\left(\mathbf{F}_{\mathfrak{p}}\right) \rightarrow \mathfrak{a}^{\vee}
$$

par la relation

$$
\forall \chi \in X^{*}(M), \quad \forall z \in Z_{M}\left(\mathbf{F}_{\mathfrak{p}}\right), \quad|\chi(z)|_{\mathfrak{p}}=q^{\left\langle H_{P, \mathfrak{p}}(z), \chi\right\rangle}
$$

et $H_{P}: M\left(\boldsymbol{A}_{F}\right) \rightarrow \mathfrak{a}^{\vee}$ est défini comme la somme des morphisme $H_{P, \mathfrak{p}}$. On étend $H_{P}$ en une application de $G\left(\boldsymbol{A}_{F}\right)$ dans $\mathfrak{a}^{\vee}$ de la manière suivante :

$$
\forall n \in N\left(\boldsymbol{A}_{F}\right), \quad \forall m \in M\left(\boldsymbol{A}_{F}\right), \quad \forall k \in K, \quad H_{P}(n m c)=H_{P}(c) .
$$

Pour tout sous-groupe compact ouvert $K^{\prime}$ de $K$, on notera $\mathscr{C}^{0}\left(P, K^{\prime}\right)$ l'ensemble des fonctions continues

$$
\varphi: P\left(\boldsymbol{A}_{F}\right) \backslash G\left(\boldsymbol{A}_{F}\right) \rightarrow \mathbf{C}
$$

telles que $\varphi$ soit $K^{\prime}$-finie à droite (i.e. l'espace vectoriel engendré par les translatés de $\varphi$ par les éléments de $K^{\prime}$ est de dimension finie). Si $\varphi \in \mathscr{C}^{0}\left(P, K^{\prime}\right)$ et $\xi \in \mathfrak{a}$, on définit

$$
\forall g \in G\left(\boldsymbol{A}_{F}\right), \quad T_{\xi} \varphi(g)=q^{\left\langle H_{P}(g), \xi\right\rangle} \varphi(g) .
$$


Définition 4.1.2. Si $\varphi \in \mathscr{C}^{0}\left(P, K^{\prime}\right)$ et $\xi \in \mathfrak{a}$, la série d'Eisenstein associée à $\varphi$ et $\xi$ est définie par

$$
\forall g \in G\left(\boldsymbol{A}_{F}\right), \quad E_{P}^{G}(\varphi, \xi, g)=\sum_{\gamma \in P(F) \backslash G(F)} T_{\xi+\rho_{P}} \varphi(\gamma g) .
$$

D'après un lemme de Godement [Mo1 §2.2.2, lemme, p. 118] cette série converge uniformément sur les sous-ensembles compacts de $G\left(\boldsymbol{A}_{F}\right)$ dés que $\operatorname{Re}\left(\xi-\rho_{P}\right)$ appartient à $C_{P}$ où $C_{P}$ est la chambre de Weyl définie par

$$
\forall \alpha \in{ }_{F} \Delta-{ }_{F} I, \quad(\lambda, \check{\alpha}) \geqslant 0 .
$$

On notera $E_{P}^{G}(\xi, \cdot)$ la fonction $E_{P}^{G}(1, \xi, \cdot)$.

Proposition 4.1.1. La série définissant la fonction zêta des hauteurs $\zeta_{V, \boldsymbol{H}}(s)$ coüncide avec celle définissant $E_{P}^{G}\left(s-\rho_{P}, e\right)$.

Démonstration. Par [Bo proposition 20.5], l'application $\pi: G(F) \rightarrow V(F)$ est surjective. IL suffit donc de vérifier que pour tout élément $g$ de $G(F)$, on a

$$
\boldsymbol{H}_{K}(\chi)(\pi(g))=q^{\left\langle H_{P}(g), \chi\right\rangle} .
$$

On écrit donc $g=n m k$ avec $n \in N\left(\boldsymbol{A}_{F}\right), m \in M\left(\boldsymbol{A}_{F}\right)$ et $k \in K$. Soit $s$ une section de $L_{\chi}$ sur un voisinage ouvert $U$ de $\pi(g)$, non nulle en $\pi(g)$ et correspondant à un élément $\tilde{s}$ de $\Gamma\left(G, \mathscr{O}_{G}\right)$. Par définition on a

$$
\boldsymbol{H}_{K}(\chi)(\pi(g))=\prod_{\mathfrak{p} \in M_{F}}\|\boldsymbol{s}(\pi(g))\|_{\mathfrak{p}}=\prod_{\mathfrak{p} \in M_{F}}\left|\tilde{\boldsymbol{s}}\left(k_{\mathfrak{p}}\right)\right|_{\mathfrak{p}}^{-1} .
$$

Mais par la formule du produit $\prod_{\mathfrak{p} \in M_{F}}|\tilde{s}(g)|_{\mathfrak{p}}=1$ et donc

$$
\left(\prod_{\mathfrak{p} \in M_{F}}\left|\chi\left(m_{\mathfrak{p}} n_{\mathfrak{p}}\right)\right|_{\mathfrak{p}}\right) \prod_{\mathfrak{p} \in M_{F}}\left|\tilde{s}\left(k_{\mathfrak{p}}\right)\right|_{\mathfrak{p}}=1 .
$$

On en déduit les égalités

$$
\boldsymbol{H}_{K}(\chi)(\pi(g))=\prod_{\mathfrak{p} \in M_{F}}\left|\chi\left(m_{\mathfrak{p}}\right)\right|_{\mathfrak{p}}=\prod_{\mathfrak{p} \in M_{F}} q^{\left\langle H_{P, \mathfrak{p}}(g), \chi\right\rangle}=q^{\left\langle H_{P}(g), \chi\right\rangle} .
$$

Corollaire 4.1.2. La fonction zêta des hauteurs $\zeta_{V, \boldsymbol{H}_{K}}$ converge absolument dans le cône ouvert

$$
\omega_{V}^{-1}+\overparen{\circ} \stackrel{\circ}{C_{\text {eff }}(V)}+i \operatorname{Pic} V \otimes \mathbf{R}
$$

et s'étend en une fonction méromorphe sur $\mathbf{C}$ qui admet une expression rationnelle en des puissance de $q$.

Démonstration. D'après [BoTi1] §12.12], on a

$$
\forall \alpha, \alpha^{\prime} \in{ }_{F} \Delta, \quad \alpha \neq \alpha^{\prime} \Rightarrow\left\langle\sum_{\beta \in j^{-1}(\alpha)} \varpi_{\beta}, \check{\alpha}\right\rangle>0 \quad \text { et }\left\langle\sum_{\beta \in j^{-1}(\alpha)} \varpi_{\beta}, \check{\alpha}^{\prime}\right\rangle=0
$$

Par conséquent le cône $C_{P}$ coïncide d'après 3.3.1 avec le cône $C_{\text {eff }}(V)$. La première assertion résulte donc de [Mo1] lemma, p. 118] mentionné cité ci-dessus. La seconde es un résultat de Morris [Mo2, §6.6, lemma, p. 1164] 
4.2. Ordre du pôle au sommet du cône. L'objectif de ce paragraphe est de montrer le résultat suivant :

Proposition 4.2.1. Le lieu des singularités de la fonction $\zeta_{V, \boldsymbol{H}_{K}}$ au voisinage du point $s=\omega_{V}^{-1}$ coüncide avec la réunion es hyperplans

$$
\left\langle\check{\alpha}, \lambda-\omega_{V}^{-1}\right\rangle=0
$$

pour $\alpha \in{ }_{F} \Delta-{ }_{F} I$, chacun de ces hyperplans intervenant avec une multiplicité au plus égale à un.

Remarques 4.2.2. (i) Nous verrons plus loin que la multiplicité est en réalité exactement égale à un.

(ii) Le fait que les singularités soient hyperplanes résulte de la proposition 4.1.1 et de [Mo2, §6.6, lemma, p. 1164].

Comme dans [FMT §2] le principe de la démonstration est de considérer la fibration

$$
P_{0} \backslash P \rightarrow P_{0} \backslash G \rightarrow V,
$$

d'exprimer le terme constant des séries d'Eisenstein correspondant à $P_{0} \backslash P$ et $P_{0} \backslash G$ en termes des opérateurs d'entrelacements. Comme dans Harder [Har p. 278] ou Morris [Mo1, §4.3.4], l'étude des singularités des séries d'Eisenstein se réduit alors à la description de celles des opérateurs d'entralecemant qui se déduisent des équations fonctionnelles et du cas de l'opérateur associé à une réflexion.

Notations 4.2.1. Pour tout $\varphi$ de $\mathscr{C}^{0}\left(P_{0}, K^{\prime}\right)$ et $\xi$ de a, la série d'Eisenstein partielle $E_{P_{0}}^{P}$ est définie par

$$
E_{P_{0}}^{P}(\varphi, \xi, g)=\sum_{\gamma \in P_{0}(F) \backslash P(F)} T_{\xi+\rho_{P_{0}}} \varphi(\gamma g) .
$$

Pour tout $w \in{ }_{F} W$ représenté par un élément $w^{\prime}$ de $\mathscr{N}_{G}(S)(k)$, la fonction $C(w, \xi) \varphi$ est définie par

$$
\forall g \in G\left(\boldsymbol{A}_{F}\right), \quad C(w, \xi) \varphi(g)=\int_{w^{\prime} N_{0}\left(\boldsymbol{A}_{F}\right) w^{\prime-1} \cap N_{0}\left(\boldsymbol{A}_{F}\right) \backslash N_{0}\left(\boldsymbol{A}_{F}\right)} q^{\left\langle H_{P_{0}}\left(w^{\prime-1} n g\right), \xi+\rho_{P_{0}}\right\rangle} \varphi\left(w^{\prime-1} n g\right) \mathrm{d} n
$$

où pour tout groupe unipotent $U$ sur $F$, la mesure de Haar sur $U\left(\boldsymbol{A}_{F}\right)$ est normalisée par

$$
\int_{U(F) \backslash U\left(\boldsymbol{A}_{F}\right)} \mathrm{d} u=1 .
$$

On note également $E_{P_{0}}^{P}(\xi, g)=E_{P_{0}}^{P}(1, \xi, g)$ et $c(w, \xi)=(C(w, \xi) 1)(e)$.

Remarque 4.2.3. Par [Mo1 §2.4.8, theorem, p. 134] et [Mo1 §4.3.1, p. 167], on a les équations fonctionnelles

$$
c\left(w_{1}, w_{2} \xi\right) c\left(w_{2}, \xi\right)=c\left(w_{1} w_{2}, \xi\right)
$$

et

$$
c(w, \lambda) E_{P_{0}}^{G}(w \lambda, g)=E_{P_{0}}^{G}(\lambda, g) .
$$

En outre, par définition, pour tout $\xi^{\prime}$ de $\mathfrak{a}$ invariant par $w$ on a

$$
c\left(w, \xi+\xi^{\prime}\right)=c(w, \xi)
$$

Lemme 4.2.4. Si $w \in{ }_{F} W$, le lieu des singularités de la fonction $c(w, \cdot)$ au voisinage $d u$ point $\lambda=\rho_{P_{0}}$ est égale à la réunion des hyperplans

$$
\left\langle\check{\alpha}, \lambda-\rho_{P_{0}}\right\rangle
$$

où $\alpha$ décrit l'ensemble $\left\{\alpha \in{ }_{F} \Delta \mid w \alpha<0\right\}$. La multiplicité de chacun de ces hyperplans est exactement égale à un. 
Remarque 4.2.5. Dans le cas où $G$ est un groupe de Chevalley, ce résultat découle immédiatement de ceux de Harder [Har, p. 278].

Démonstration. Comme dans [FMT] p. 429], si $w=s_{\alpha}$ avec $\alpha \in{ }_{F} \Delta$ il résulte de 4.2.3 que l'hyperplan défini par

$$
\left\langle\check{\alpha}, \lambda-\rho_{P_{0}}\right\rangle=0
$$

contient le lieu singulier de $c\left(s_{\alpha}, c d o t\right)$ au voisinage de $\rho_{P_{0}}$. Le fait que ce pôle soit au plus de multiplicité un résulte de [Mo1 \$3.5.2, theorem(i)]. Pour montrer que c'est effectivement un pôle, il suffit d'écrire $c\left(s_{\alpha}, \cdot\right)$ comme produit de facteurs locaux (cf. aussi la démonstration du théorème 4.3.1.

En général, on écrit

$$
w=s_{\alpha_{1}} \ldots s_{\alpha_{q}}
$$

avec $\alpha_{i} \in{ }_{F} \Delta$ et $q$ minimal. On pose

$$
w_{j}=s_{\alpha_{j+1}} \ldots s_{\alpha_{q}} .
$$

Alors l'équation fonctionnelle 4.2.1) fournit l'égalité

$$
c(w, \lambda)=c\left(s_{\alpha_{1}}, w_{1} \lambda\right) \ldots c\left(s_{\alpha_{q}}, \lambda\right) .
$$

Mais par [Bki] Ch. VI, \$1.1.6, corollaire 2, p. 158], on a que $w_{j}^{-1} \alpha_{j}<0$ et que $\{\alpha \in$ $\left.{ }_{F} \Phi^{+} \mid w \alpha<0\right\}$ coïncide avec l'ensemble $\left\{w_{j}^{-1}, 1 \leqslant j \leqslant q\right\}$. Il résulte alors de [FMT] $\S 8$, sublemma, p. 430] que

$$
\left\langle\alpha_{j}, w_{j} \rho_{P_{0}}\right\rangle \geqslant\left\langle\check{\alpha}_{j}, \rho_{P_{0}}\right\rangle
$$

avec égalité uniquement si $w_{j}^{-1} \alpha_{j} \in{ }_{F} \Delta$. Comme dans [FMT], on déduit alors du cas qui précède que $c\left(s_{\alpha_{j}}, w_{j} \cdot\right)$ est régulier au voisinage de $\rho_{P_{0}}$ sauf si $w_{j}^{-1} \alpha_{j} \in{ }_{F} \Delta$ auquel cas la singularité est contenu dans l'hyperplan

$$
\left\langle w_{j}^{-1} \alpha_{j}, \lambda-\rho_{P_{0}}\right\rangle=0
$$

qui est de multiplicité un.

Fin de la démonstration de la proposition [4.2.1] Il résulte de [Mo1, §4.3.4], que les singularités de $E_{P_{0}}^{G}(\cdot, g)$ sont dominées par celles de $c\left({ }_{F} w_{F} \Delta, \cdot\right)$. le résultat est donc démontré dans le cas où $P=P_{0}$. Dans le cas général, pour tout $\xi$ de

$$
\rho_{P_{0}}+C_{P_{0}}+i X^{*}(S) \otimes \mathbf{R}
$$

le terme constant de $E_{P_{0}}^{P}$ est donné, d'après la démonstration de [Mo1] \$2.3.1, lemma, p. 122], par la formule

$$
\int_{N_{0}(\mathbf{F}) \backslash N_{0}\left(\boldsymbol{A}_{F}\right)} E_{P_{0}}^{P}(\xi, n g) \mathrm{d} n=\sum_{w ! i n_{F} W_{F}} c(w, \xi) q^{\left\langle H_{P_{0}}(g), w \xi+2 \rho_{P}\right\rangle}
$$

En prenant les systèmes d'Eisenstein résiduels successifs (cf [Mo1, exemple 3.11.2, p. $1130-1132])$ on obtient pour tout $\xi$ de $\mathfrak{a}$ la relation

$$
\lim _{\substack{\lambda \rightarrow 0 \\ \lambda \in \mathfrak{a}^{\perp}}}\left(\prod_{\alpha \in F^{\prime}}\langle\check{\alpha}, \lambda\rangle\right) E_{P_{0}}^{P}\left(\lambda+\xi+\rho_{P_{0}}, g\right)=C_{P} q^{\left\langle H_{P}(g), \xi+2 \rho_{P}\right\rangle}
$$

où $\mathfrak{a}^{\perp}=\left\{\lambda \in X^{*}(S) \otimes \mathbf{R} \mid \lambda_{\left.\right|_{F} S_{I}}=0\right\}$ et la constante $C_{P}$ est définie par

$$
C_{P}=\lim _{\lambda \rightarrow \rho_{P_{0}}}\left(\prod_{\alpha \in \in_{F} I}\left\langle\check{\alpha}, \lambda-\rho_{P_{0}}\right\rangle\right) c\left({ }_{F} w_{F} I, \lambda\right) .
$$

En sommant sur $P(F) \backslash G(F)$, on obtient

$$
\lim _{\substack{\lambda \rightarrow 0 \\ \lambda \in \mathfrak{a}^{\perp}}}\left(\prod_{\alpha \in F I}\langle\check{\alpha}, \lambda\rangle\right) E_{P_{0}}^{G}\left(\lambda+\xi+\rho_{P_{0}}, g\right)=C_{P} E_{P}^{G}\left(\xi+\rho_{P}, g\right)
$$

et l'assertion pour $E_{P}^{G}$ découle de celle pour $E_{P_{0}}^{G}$ et du fait que $C_{P} \neq 0$. 
Remarque 4.2.6. Il découle de la démonstration précédente que que

$$
\lim _{\xi \rightarrow 0}\left(\prod_{\alpha \in \Delta_{0}-{ }_{F} I}\langle\check{\alpha}, \xi\rangle\right) E_{P}^{G}\left(\xi+\rho_{P}, g\right)=C_{G} / C_{P}
$$

et donc par la proposition 4.1 .1

$$
\lim _{s \rightarrow 1}(s-1)^{\mathrm{rg} \operatorname{Pic} V} \zeta_{V, \boldsymbol{H}_{K}}\left((s-1) \omega_{V}^{-1}\right)=\left(\prod_{\alpha \in \in_{F} \Delta_{F}}\left\langle\check{\alpha}, 2 \rho_{P}\right\rangle^{-1}\right) \frac{C_{G}}{C_{P}}
$$

4.3. Valeur de la constante. Par la remarque précédente, pour clôre la démonstration, il suffit de comparer $C_{G} / C_{P}$ et $\theta_{H}^{*}(V)$ ce qui redémontrera du même coup que $C_{G} / C_{P}$ est une constante non nulle et que les multiplicités des hyperplans $\langle\check{\alpha}, \xi\rangle=0$ sont bien égales à un.

Théorème 4.3.1. On a la relation

$$
\left(\prod_{\alpha \in{ }_{F} \Delta_{F^{I}}}\left\langle\check{\alpha}, 2 \rho_{P}\right\rangle\right) \frac{C_{G}}{C_{P}}=\theta_{H}^{*}(V)
$$

Pour montrer ce résultat, il nous faut d'abord écrire l'opérateur d'entrelacement comme produit d'opérateurs locaux que nous allons maintenant définir.

Notations 4.3.1. Pour toute place $\mathfrak{p}$ de $F$, on note ${ }_{F_{\mathfrak{p}}} S$ un tore scindé maximal de $G_{F_{\mathfrak{p}}}$ tel que

$$
{ }_{F} S_{F_{\mathfrak{p}}} \subset{ }_{F_{\mathrm{p}}} S \subset P_{0 F_{\mathfrak{p}}},
$$

on note ${ }_{F_{\mathrm{p}}} P_{0}$ un sous-groupe parabolique minimal de $G_{F_{\mathrm{p}}}$ tel que

$$
{ }_{F_{\mathfrak{p}}} S \subset{ }_{F_{\mathfrak{p}}} P_{0} \subset P_{0 F_{\mathfrak{p}}}
$$

et ${ }_{F_{\mathfrak{p}}} N$ son radical unipotent. Quitte à modifier le choix de certains des compacts $K_{\mathfrak{p}}$, on peut supposer que

$$
G\left(F_{\mathfrak{p}}\right)={ }_{F_{\mathfrak{p}}} P_{0}\left(F_{\mathfrak{p}}\right) K_{\mathfrak{p}}
$$

On note $F_{\mathfrak{p}} \Phi$ le système de ${ }_{F_{\mathfrak{p}}} S$ dans $G_{F_{\mathfrak{p}}}$ et ${ }_{F_{\mathfrak{p}}} \Delta$ la base de ${ }_{F_{\mathfrak{p}}} \Phi$ correspondant à ${ }_{F_{\mathfrak{p}}} P_{0}$.

Si $J$ est une partie de ${ }_{F_{\mathrm{p}}} \Delta$, od définit comme précédemment le sous groupe parabolique ${ }_{F_{\mathfrak{p}}} P_{J}$, l'algèbre de Lie $F_{F_{\mathfrak{p}}} \mathfrak{r}_{J}$, le $\mathbf{C}$-espace vectoriel ${ }_{F_{\mathfrak{p}}} \mathfrak{a}_{J}$, l'élément ${ }_{F_{\mathfrak{p}}} w_{J}$ du groupe de Weyl le caractère $\rho_{F_{\mathfrak{p}}} P_{J}$ et la fonction $H_{F_{\mathfrak{p}} P_{J}, \mathfrak{p}}$. On se donne en outre des bases des sousespaces propres de $F_{F_{\mathrm{p}}} \mathfrak{r}_{\emptyset}$ pour l'action de ${ }_{F_{\mathrm{p}}} S$ de sorte que pour toute partie $J$ de ${ }_{F} \Delta$, l'isomorphisme de $F$-espaces vectoriels

$$
\Lambda^{\operatorname{dim}}{ }_{F} \mathfrak{r}_{J}\left({ }_{F} \mathfrak{r}_{J} \otimes \mathbf{F}_{\mathfrak{p}}\right) \stackrel{\sim}{\longrightarrow} \mathbf{F}_{\mathfrak{p}}
$$

induit par ces bases coïncide avec celui induit par les bases choisies sur $F$.

Si $U$ est un $F_{\mathfrak{p}}$ groupe unipotent de ${ }_{F_{\mathfrak{p}}} P_{\emptyset}$ ou de son opposé, alors ces bases définissent un isomorphisme de variété de $U$ sur $\mathbf{A}_{F_{\mathfrak{p}}}^{\operatorname{dim} U}$, ce qui permet de normaliser la mesure de Haar sur $U\left(F_{\mathfrak{p}}\right)$.

Quitte à modifier à nouveau certains des $K_{\mathfrak{p}}$, on peut fixer pour tout $w$ de ${ }_{F} W$ des représentants $\tilde{w}$ appartenant à $\mathscr{N}_{G}(S)(F) \cap K$.

Définition 4.3.2. Pour tout $\lambda$ de ${ }_{F} \mathfrak{a}_{0}$, et tout $w$ de ${ }_{F} W$, on considère

$$
{ }_{F} c_{\mathfrak{p}}(w, \lambda)=\int_{\left[\tilde{w}_{F} N\left(F_{\mathfrak{p}}\right) \tilde{w}^{-1} \cap_{F} N\left(F_{\mathfrak{p}}\right)\right] \backslash_{F} N\left(F_{\mathfrak{p}}\right)} \exp \left(\left\langle H_{P_{0}, \mathfrak{p}}\left(\tilde{w}^{1} n\right), \lambda+\rho_{P_{0}}\right\rangle\right) \mathrm{d} n_{\mathfrak{p}}
$$

Remarque 4.3.2. Le quotient $C_{G} / C_{P}$ se met alors sous la forme

$$
\frac{C_{G}}{C_{P}}=\frac{1}{q^{(g-1) \operatorname{dim} V}} \lim _{\lambda \rightarrow 0} \prod_{\alpha \in \in_{F}-I}\langle\check{\alpha}, \lambda\rangle \prod_{\mathfrak{p} \in M_{F}} \frac{{ }_{F} c_{\mathfrak{p}}\left({ }_{F} w_{F} \Delta, \lambda+\rho_{P_{0}}\right)}{{ }_{F} c_{\mathfrak{p}}\left({ }_{F} w_{I}, \lambda+\rho_{P_{0}}\right)}
$$


Définition 4.3.3. Pour tout $\lambda$ de ${ }_{F_{\mathfrak{p}}} \mathfrak{a}_{0}$ et tout $w$ de ${ }_{F_{\mathfrak{p}}} W$ se relevant en $w^{\prime} \in K_{\mathfrak{p}}$

$$
\begin{aligned}
& c_{\mathfrak{p}}(w, \lambda)=\int \exp \left(\left\langle H_{F_{\mathfrak{p}} P_{0}, \mathfrak{p}}\left(w^{\prime-1}, \lambda+\rho_{F_{\mathfrak{p}} P}\right\rangle\right) \mathrm{d} n_{\mathfrak{p}}\right. \\
& {\left[w_{F_{\mathfrak{p}}}^{\prime} N\left(F_{\mathfrak{p}}\right) w^{\prime-1} \cap_{F_{\mathfrak{p}}} N\left(F_{\mathfrak{p}}\right)\right] \backslash_{F_{\mathfrak{p}}} N\left(F_{\mathfrak{p}}\right)}
\end{aligned}
$$

Remarque 4.3.3. Casselman donne dans [Cas] une expression explicite pour $c_{\mathfrak{p}}(w, \lambda)$ en reliant ${ }_{F} c_{\mathfrak{p}}(w, \lambda)$ à ce terme, on obtiendra une expression explicite pour ce dernier.

Lemme 4.3.4. Le volume de la variété à la place $\mathfrak{p}$ vérifie

$$
\omega_{\mathfrak{p}}\left(V\left(F_{\mathfrak{p}}\right)\right)=\frac{{ }_{F} c_{\mathfrak{p}}\left(w_{F} \Delta, \rho_{P_{0}}\right)}{{ }_{F} c_{\mathfrak{p}}\left(w_{F} I, \rho_{P_{0}}\right)}
$$

Démonstration. Ceci résulte immédiatement de la démonstration du lemme 6.2.7 dans [Pe1].

Lemme 4.3.5. Avec les notations précédentes on a la relation

$$
\frac{{ }_{F} c_{\mathfrak{p}}\left({ }_{F} w_{F} \Delta, \lambda+\rho_{P_{0}}\right)}{{ }_{F} c_{\mathfrak{p}}\left({ }_{F} w_{F} I, \lambda+\rho_{P_{0}}\right)}=\frac{c_{\mathfrak{p}}\left(w_{F_{\mathfrak{p}}}, \operatorname{Res} \lambda+\rho_{F_{\mathfrak{p}} P}\right)}{c_{\mathfrak{p}}\left(w_{F_{\mathfrak{p}} I}, \operatorname{Res} \lambda+\rho_{F_{\mathfrak{p}} P}\right)}
$$

où ${ }_{F_{\mathfrak{p}}} I$ désigne la partie de ${ }_{F_{\mathfrak{p}}} \Delta$ correspondant à $P_{F_{\mathfrak{p}}}$.

Démonstration. Ce lemme se montre comme le lemme 6.2.8 de [Pe1].

Notation 4.3.4. On note

$$
\lambda_{P_{0}}=\sum \alpha \in j^{-1}\left({ }_{F} \Delta\right) \varpi_{\alpha} \in \operatorname{Pic}\left(V_{0}\right) \stackrel{\sim}{\longrightarrow} X^{*}\left(P_{0}\right)_{F} \subset \mathfrak{a}_{0} .
$$

Lemme 4.3.6. Le produit

$$
\prod_{\mathfrak{p} \in M_{F}} L_{\mathfrak{p}}(s, \operatorname{Pic} \bar{V})^{-1} \frac{c_{\mathfrak{p}}\left({ }_{F_{\mathfrak{p}}} w_{F_{\mathfrak{p}} \Delta},(s-1) \operatorname{Res} \lambda_{F} P+\rho_{F_{\mathfrak{p}} P_{0}}\right)}{c_{\mathfrak{p}}\left({ }_{F_{\mathfrak{p}}} w_{F} I,(s-1) \operatorname{Res} \lambda_{F} P+\rho_{F_{\mathfrak{p}} P_{0}}\right)}
$$

converge absolument au voisinage de $s=1$.

Démonstration. Comme dans la démonstration du lemme 6.2.12 de [Pe1], cela résulte de l'expression explicite donnée par Casselmann de $c_{\mathfrak{p}}(w, \chi)$.

Lemme 4.3.7. La constante $\alpha^{*}(V)$ est donnée par la formule

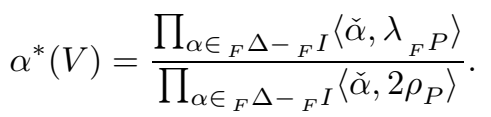

Démonstration. Ceci résulte immédiatement de la description de $C_{\mathrm{eff}}(V)$ donnée par l'expression 3.3.1 et du fait, déja indiqué en 4.1.1 que la matrice de changement de base passant de $(\check{\alpha})_{\alpha \in{ }_{F} \Delta}$ à la base duale de $\left(\sum_{\beta \in j^{-1}(\alpha)} \varpi_{\beta}\right)_{\alpha \in{ }_{F} \Delta}$ est diagonale.

Démonstration du théorème 4.3.1 Par a formule 4.3.2, on a

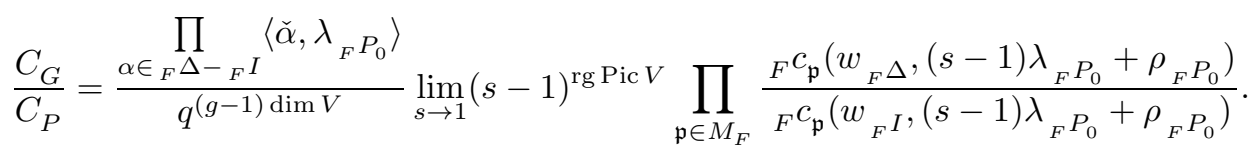

Pour tout $\mathfrak{p}$ de $M_{F}$, on note $\lambda_{\mathfrak{p}}(s)=L_{\mathfrak{p}}(s, \operatorname{Pic} \bar{V})$ et le quotient $C_{G} / C_{P}$ s'écrit

$$
\begin{aligned}
& \frac{\prod_{\alpha \in{ }_{F} \Delta-{ }_{F} I}\left\langle\check{\alpha}, \lambda_{F} P_{0}\right\rangle}{q^{(g-1) \operatorname{dim} V}} \lim _{s \rightarrow 1}\left[(s-1)^{\operatorname{rg} \operatorname{Pic} V} L(s, \operatorname{Pic} \bar{V})\right.
\end{aligned}
$$

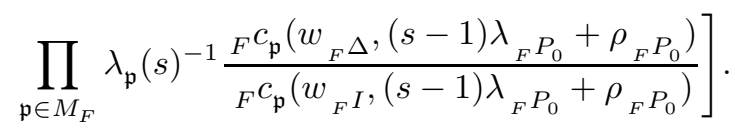


par les lemmes 4.3.5 et 4.3.6 le produit du bas converge absolument au voisinage de 1 et le quotient $C_{G} / C_{P}$ se met sous la forme

$$
\begin{aligned}
& \prod_{\alpha \in F_{F} \Delta-{ }_{F} I}\left\langle\check{\alpha}, \lambda_{F} P_{0}\right\rangle \frac{\lim _{s \rightarrow 1}(s-1)^{\operatorname{rg} \operatorname{Pic} V} L(s, \operatorname{Pic} \bar{V})}{q^{(g-1) \operatorname{dim} V}} \prod_{\mathfrak{p} \in M_{F}} \lambda_{\mathfrak{p}}(1)^{-1} \frac{{ }_{F} c_{\mathfrak{p}}\left(w_{F} \Delta, \rho_{F} P_{0}\right)}{{ }_{F} c_{\mathfrak{p}}\left(w_{F} I, \rho_{F} P_{0}\right)} \\
= & \prod_{\alpha \in F_{F} \Delta-{ }_{F} I}\left\langle\check{\alpha}, \lambda_{F} P_{0}\right\rangle \tau_{H_{K}}(V)
\end{aligned}
$$

ce qui conclut la démonstration.

Je tiens à remercier Laure Blasco pour ses précieuses indications.

\section{RÉFÉRENCES}

[BM] V. V. Batyrev et Y. I. Manin, Sur le nombre des points rationnels de hauteur bornée des variétés algébriques, Math. Ann. 286 (1990), 27-43.

[BT1] V. V. Batyrev and Y. Tschinkel, Rational points of bounded height on compactifications of anisotropic tori, Internat. Math. Res. Notices 12 (1995), 591-635.

[BT2] _ Tamagawa numbers of polarized algebraic varieties, Nombre et répartition de points de hauteur bornée, Astérisque, vol. 251, SMF, Paris, 1998, pp. 299-340.

[BT3] - Manin's conjecture for toric varieties, J. Algebraic Geom. 7 (1998), $\mathrm{n}^{\mathrm{O}}$ 1, 15-53.

[Bo] A. Borel, Linear algebraic groups (Second enlarged edition), Graduate Texts in Math., vol. 126, SpringerVerlag, Berlin, Heidelberg and New York, 1991.

[BoTi1] A. Borel et J. Tits, Groupes réductifs, Publ. Math. I.H.E.S. 27 (1965), 55-150.

[BoTi2] —_ Compléments à l'article : « groupes réductifs 》, Publ. Math. I.H.E.S. 41 (1972), 253-276.

[Bki] N. Bourbaki, Groupes et algèbres de Lie. Chap. 4, 5 et 6, Masson, Paris, 1981.

[Bou1] D. Bourqui, Fonction zêta des hauteurs des surfaces de Hirzebruch dans le cas fonctionnel, J. of Number Theory 94 (2002), 343-358.

[Bou2] __ Fonction zêta des hauteurs des variétés toriques déployées dans le cas fonctionnel (2002).

[Bou3] _ Points de hauteur bornée sur les variétés toriques en caractéristique positive, prépublication (2003).

[Cas] W. Casselman, The unramified principal series of $\mathfrak{p}$-adic groups I. The spherical function, Compositio Math. 40 (1980), n⿳0 3, 387-406.

[CTS] J.-L. Colliot-Thélène et J.-J. Sansuc, La descente sur les variétés rationnelles, II, Duke Math. J. 54 (1987), $\mathrm{n}^{\mathrm{O}} 2,375-492$.

[Del] P. Deligne, La conjecture de Weil I., Publ. Math. I.H.E.S. 43 (1973), 273-307.

[Dem] M. Demazure, Sous-groupes paraboliques des groupes réductifs, Schémas en groupes III (Séminaire de géométrie algébrique du Bois-Marie 1962/64 (SGA3)), Lect. Notes in Math., n⿳0 153, Springer-Verlag, Berlin, Heidelberg and New York.

[EGA] J. Dieudonné et A. Grothendieck, Eléments de géométrie algébrique, Publ. Math. I.H.E.S., vol. 4, 8, 11, 17, 20, 24, 28 et 32, 1960-67.

[Dr] P. K. J. Draxl, L-Funktionen algebraischer Tori, J. Number Theory 3 (1971), 444-467.

[FMT] J. Franke, Y. I. Manin, and Y. Tschinkel, Rational points of bounded height on Fano varieties, Invent. Math. 95 (1989), 421-435.

[Gr1] A. Grothendieck, Géométrie formelle et géométrie algébrique, Séminaire Bourbaki 11-ème année, $1958 / 59, \mathrm{n}^{\mathrm{O}} 182$

[Gr2] Le groupe de Brauer III : Exemples et compléments, Dix exposés sur la cohomologie des schémas, Adv. Stud. Pure Math., vol. 3, North-Holland, Amsterdam et Masson, Paris, 1968, pp. 88-187.

[Gr3] Le groupe de Brauer II : Théorie cohomologique, Dix exposés sur la cohomologie des schémas, Adv. Stud. Pure Math., vol. 3, North-Holland, Amsterdam et Masson, Paris, 1968, pp. 67-87.

[Har] G. Harder, Chevalley groups over function fields and automorphic forms, Ann. of Math. 100 (1974), 249-306.

[Ha] R. Hartshorne, Algebraic geometry, Graduate Texts in Math., vol. 52, Springer-Verlag, Berlin, Heidelberg and New York, 1977. 
[Hs] Liang chung Hsia, On the dynamical height zeta functions, J. Number Theory 63 (1997), 146-169.

[Ke] G. R. Kempf, Algebraic representations of reductive groups, Proc. of the Internat. Congress of Math. (Helsinki, 1978), Acad. Sci. Fennica, Helsinki, 1980, pp. 575-577.

[Kö] M. Köcher, Positivitätsbereiche im $\mathbf{R}^{n}$, Amer. J. Math. 79 (1957), 575-596.

[LY] K. F. Lai and K. M. Yeung, Rational points in flag varieties, J. of Number Theory 95 (2002), $142-149$.

[La] S. Lang, Fundamentals of diophantine equations, Springer-Verlag, Berlin, Heidelberg and New York, 1983.

[Mo1] L. E. Morris, Eisenstein series for reductive groups over global function fields I. The cusp form case, Can. J. Math. 34 (1982), 91-168.

[Mo2] Eisenstein series for reductive groups over global function fields II. The general case, Can. J. Math. 34 (1982), 1112-1182.

[Oda] T. Oda, Convex bodies and algebraic geometry, Ergebnisse der Mathematik und ihrer Grenzgebiete, 3. Folge, vol. 15, Springer-Verlag, Berlin, Heidelberg and New York, 1988.

[Pe1] E. Peyre, Hauteurs et mesures de Tamagawa sur les variétés de Fano, Duke Math. J. 79 (1995), $\mathrm{n}^{\mathrm{O}}$ 1, $101-218$.

[Pe2] Terme principal de la fonction zêta des hauteurs et torseurs universels, Nombre et répartition de points de hauteur bornée, Astérisque, vol. 251, SMF, Paris, 1998, pp. 259-298.

[Pe3] Torseurs universels et méthode du cercle, Rational points on algebraic varieties, Progress in Math., vol. 199, Birkhaüser, Basel, 2001, pp. 221-274.

[Ro] M. Rosenlicht, Toroidal algebraic groups, Proc. Amer. Math. Soc. 12 (1961), 984-988.

[Sa] P. Salberger, Tamagawa measures on universal torsors and points of bounded height on Fano varieties, Nombre et répartition de points de hauteur bornée, Astérisque, vol. 251, SMF, Paris, 1998, pp. 91-258.

[San] J.-J. Sansuc, Groupe de Brauer et arithmétique des groupes algébriques linéaires sur un corps de nombres, J. Reine Angew. Math. 327 (1981), 12-80.

[Se1] J.-P. Serre, Facteurs locaux des fonctions zêta des variétés algébriques (définitions et conjectures), Séminaire Delange-Pisot-Poitou 11-ème année, 1969/70, n ${ }^{\mathrm{o}} 19$.

[Se2] Valeurs propres des endomorphismes de Frobenius (d'après P. Deligne), Séminaire Bourbaki 26-ème année, 1973/74, n 0446.

[Se3] L Lectures on the Mordell-Weil theorem, Aspects of Mathematics, vol. E15, Vieweg, Braunschweig, Wiesbaden, 1989.

[Si] J. H. Silverman, The theory of height functions, Arithmetic geometry (G. Cornell and J.H. Silverman, eds.), Springer-Verlag, Berlin, Heidelberg and New York, 1986, pp. 151-166.

[Tit] J. Tits, Reductive groups over local fields, (A. Borel and W. Casselman, eds.), Proc. Sympos. Pure Math., vol. 33 Part 1, AMS, Providence, 1979, pp. 29-69.

[We] A. Weil, Adèles and algebraic groups, Progress in Mathematics, vol. 23, Birkhaüser, Boston, Basel, Stuttgart, 1982.

InSTITUT Fourier, UFR DE MATHÉMATIQUES, UMR 5582, UNIVERSité DE GRENOBLE I ET CNRS, BP 74, 38402 SAINT-MARTIN D'HÈres CEDEX, FrANCE

URL: http: //www-fourier.ujf-grenoble.fr/ peyre

E-mail address: Emmanuel.Peyre@ujf-grenoble.fr 